CU-TP-1112

CLNS 04/1880

NSF-KITP-04-55

\title{
Collisions of Cosmic F- and D-strings
}

\author{
Mark G. Jackson ${ }^{1}$ \\ Department of Physics \\ Columbia Univ. \\ New York, NY 10027 \\ markj@phys.columbia.edu \\ Nicholas T. Jones ${ }^{2}$ \\ Laboratory for Elementary-Particle Physics \\ Cornell University \\ Ithaca, NY 14853 \\ nick.jones@cornell.edu \\ Joseph Polchinski \\ Kavli Institute for Theoretical Physics \\ University of California \\ Santa Barbara, CA 93106-4030, USA \\ joep@kitp.ucsb.edu
}

\begin{abstract}
Recent work suggests that fundamental and Dirichlet strings, and their $(p, q)$ bound states, may be observed as cosmic strings. The evolution of cosmic string networks, and therefore their observational signals, depends on what happens when two strings collide. We study this in string perturbation theory for collisions between all possible pairs of strings; different cases involve sphere, disk, and annulus amplitudes. The result also depends on the details of compactification; the dependence on ratios of scales is only logarithmic, but this is still numerically important. We study a range of models and parameters, and find that in most cases these strings can be distinguished from cosmic strings that arise as gauge theory solitons.
\end{abstract}

\footnotetext{
${ }^{1}$ Address after Sept. 1, 2004: Theoretical Astrophysics, Fermilab, Batavia, IL 60510

${ }^{2}$ Address after Sept. 1, 2004: University of Amsterdam
} 


\section{Introduction}

The observation of fundamental strings of cosmic size would be a spectacular window into short-distance physics. The existence of such cosmic fundamental strings in conventional Planck-scale string models is unlikely for several reasons [1]: they are unstable either to breakage or to confinement by domain walls, and even if stable they would be removed by inflation. However, in lower scale models based on large or warped compact dimensions, cosmic fundamental strings may indeed exist, as well as cosmic strings arising from D-strings and wrapped D-, NS-, and M-branes. These can be produced after inflation [2-7], and in some models they are stable or at least metastable on cosmic time scales $[8,9] .^{3}$

The observational signatures of cosmic strings depend on the detailed evolution of the string network from its creation to today [10]. This evolution in turn depends on what the strings do when they collide. For two strings of the same type, there are two obvious possibilities: they may pass simply pass through one another, or they may reconnect (intercommute) as in figure 1. Reconnection contributes to the decay of
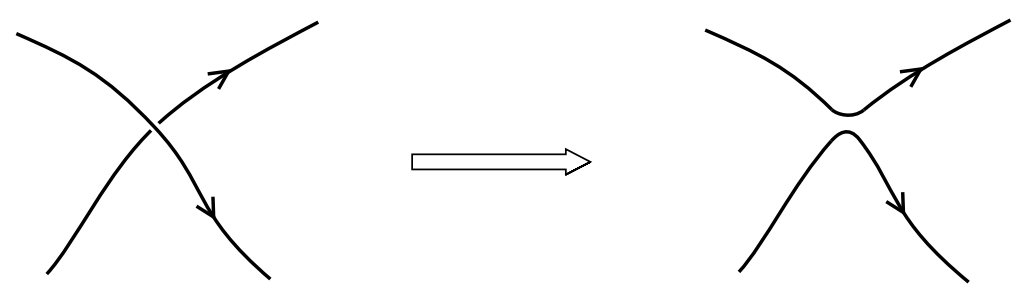

Figure 1: When two strings of the same type cross, they can reconnect (intercommute).

string networks, allowing large loops to break into smaller ones. For the usual cosmic strings, which arise as solitons (magnetic flux tubes) in gauge theories, the reconnection probability is essentially one [11]. For fundamental strings, however, the reconnection probability is of order $g_{\mathrm{s}}^{2}$, and so can be much less than one. This reduced probability will lead to an increased density of strings [12], and so to the enhancement of some signatures.

Optimistically, we may envision an era of precision cosmic string cosmology, when we will distinguish fundamental strings from solitonic strings via their intercommu-

\footnotetext{
${ }^{3}$ There is a discrepancy between refs. [8] and [9], which we will not address here.
} 
tation properties. In this paper we prepare for this happy future, by calculating the numerical factors that enter into the reconnection probability $P$. To be maximally prepared, we consider not only the fundamental F-strings, but also D-strings and bound states of $\mathrm{F}$ and D strings.

In $\S 2$ we give a primer on the properties of the $\mathrm{F}$ - and D-strings of the IIB string theory, which we hope will be broadly accessible. We also briefly discuss more general string theories. In $\S 3$ we consider F-F collisions. The basic method was developed in ref. [13], wrapping the F-strings on a large torus and using unitarity to relate the reconnection probability to a four-point tree amplitude. Here we extend the result to the supersymmetric case. In $\S 4$ we consider $\mathrm{F}-\mathrm{D}$ and $\mathrm{F}-(p, q)$ collisions, using a similar unitarity method [14]. We also show that the $\mathrm{F}-\mathrm{X}$ reconnection probability, for all string types $\mathrm{X}$, can be written in a universal form in terms of the long distance supergravity fields of X. In $\S 5$ we consider D-D collisions, using the approach first set out in ref. [15]. These are more complicated than the collisions of F-strings, but we are able to obtain useful approximate results in various regimes. In $\S 6$ we consider the collisions of vertices in the string network, including the disconnection process which is the inverse to that considered in $\S 4$ and $\S 5$.

The calculations in $\S 3$ to $\S 6$ are for toroidal compactification, where the strings are free to move in a flat higher-dimensional spacetime. In realistic compactifications one expects that all flat directions will be lifted, and the strings will sit near the minimum of a potential in the compact directions. We consider this situation in $\S 7$, and show that if the scale of the potential is somewhat less than the scale of the string tension, then it is possible to use the flat spacetime calculation in combination with an effective compactification volume arising from the quantum fluctuations of the string. The effective volume depends only logarithmically on the ratio of scales, not on powers, but nevertheless is numerically important. In $\S 8$ we bring all of our results together for some representative models. We find that $P$ in most cases is less than one, so that F- and D-strings can in principle be distinguished from gauge theory strings. In some cases $P$ is as small as $10^{-3}$, which would have a large effect on the behavior of string networks. The reconnection probability for strings of different types depends strongly on the details of the compactification and can either be rather large or essentially zero. In either case the effect on the string networks can be large, as we discuss in the conclusions. 


\section{Reconnection in field theory: a brief review}

Let us briefly review the situation with reconnection of field theory strings. Magnetic flux tubes are classical gauge theory solitons. If the gauge theory is perturbative then the string evolution is described by the classical field equations and is deterministic: for given incoming velocity and angle the strings either reconnect or they do not. In adiabatic collisions they always reconnect, because this allows the flux (and the zero of the Higgs field) to take an energetically favorable shortcut. Simulations of the classical equations show that this persists up to a center of mass velocity of around $0.9 c$ [11], above which the strings pass through one another. ${ }^{4}$ Cosmic string networks are moderately relativistic, $v \sim 0.6-0.7 c$. Only $1-2 \%$ of collisions will reach $0.9 c$ in the center of mass, so the reconnection probability $P$ is essentially one.

Of course, the simulations consider only the simplest field theory models. Consider a classical field theory with a continuous global symmetry that is unbroken in vacuum but broken in the string core. The string will have an additional collective coordinate, analogous to motion in a higher dimensional space, and two strings might avoid each other due to their separation in this coordinate [17]. This was explored in ref. [18], where it was found that $P$ remains unity at least in the moduli space limit. Ref. [18] also observes that for a broken discrete symmetry there will be $N$ types of string with self-reconnection probability $P_{\mathrm{s}}=1$ and nonself-reconnection probability $P_{\mathrm{ns}}<1$ (depending on the collision energy and barrier height). Taking for simplicity $P_{\mathrm{ns}}=0$, one gets an 'average' $P$ equal to $1 / N$.

This last model provides a nice illustration of the potential of cosmic string phenomenology. For a single string type, two things happen as $P$ is reduced: (1) the number of long strings increases, most likely as $1 / P$, so as to give the same longstring reconnection rate [19]; (2) the short-distance kinkiness of the strings increases, so that number of self-intersections increases by a factor $1 / P$, so as to give the same loop production rate per long string (as required for the network to scale [20]). With $P_{\mathrm{s}}=1, P_{\mathrm{ns}}=0$ one will have the first effect but not the second. If we are fortunate enough to see the string network directly, either through lensing or its effect on the

\footnotetext{
${ }^{4}$ We know of no good analytic determination of this crossover velocity. Ref. [16] has constructed an analytic model of both soliton and D-string scattering. This appears to have a lower crossover velocity; it would be interesting to understand the difference, but for now we assume that the simulations capture the details of the string interaction more completely.
} 
CMB, then it should be possible to distinguish this situation from a single species with $P=1 / N$ in short order.

Another possibility is gauge theory electric flux tubes [1], which would have a reconnection probability of order $1 / N_{c}^{2}$. Also, in a gauge theory with $\alpha_{\mathrm{YM}} \sim 1$, or a string theory with $g_{\text {string }} \sim 1$, the distinction between different kinds of objects

disappears and it becomes difficult to identify distinctive signatures (of course the same is true of ordinary accelerator signatures as well). However, as the example in the previous paragraph shows, the study of cosmic string networks has the potential to differentiate between seemingly similar microscopic models, even if not to resolve all degeneracies, and so we should not be too pessimistic.

\section{A primer on F- and D-strings}

We will focus primarily on the IIB string theory, both because it provides the most welldeveloped string model of inflationary cosmology [6] and because it has a potentially rich set of cosmic strings. The fundamental IIB string has a tension that we will denote $\mu_{\mathrm{F}}$, whose value might lie anywhere between the $\mathrm{TeV}$ scale and the Planck scale, though in the brane inflation models that provide much of our motivation one expects the narrower range $10^{-12}<G \mu_{\mathrm{F}}<10^{-6}[2-6]$. The string in principle oscillates in all nine spatial dimensions, but as we will discuss in $\S 7$ one expects the oscillations in the compact directions acquire nonzero world-sheet masses. It also has neutral fermionic degrees of freedom, but these will be massive as well as a consequence of supersymmetry breaking.

Besides its tension, other important properties of a cosmic string are its couplings to axions and to Standard Model fields. In the models of ref. [8], all strings are non-axionic. That is, they couple to massless potentials in ten-dimensions, but there are no light modes of these fields in the four-dimensional gauge theory. More generally, all axions in string theory are expected to have instanton-generated potentials, so that axionic strings would be confined and uninteresting as cosmic strings [1,21]. (It is conceivable that there are models where the instanton action is very small and there are interesting axionic strings.) Also in the models of ref. [8], stability of the cosmic strings requires in most cases that the strings be physically separated in the compact directions from Standard Model and other light fields, so they will be somewhat decoupled from these 
and in particular will not be superconducting.

The IIB string theory also has odd-dimensional D-branes [22], in particular Dstrings. The D-string is much like the F-string except for its tension,

$$
\mu_{\mathrm{D}}=\frac{\mu_{\mathrm{F}}}{g_{\mathrm{s}}} .
$$

The dimensionless string coupling $g_{\mathrm{s}}$ is determined by the expectation value of the dilaton field, $g_{\mathrm{s}}=e^{\Phi}$ (we are temporarily setting the $\mathrm{RR}$ scalar to zero, but will include it shortly). If the dilaton varies in the compact directions, its value at the position of the string is the relevant one. There is a special class of models in which $g_{\mathrm{s}}$ is related to observed parameters. Namely, if the Standard Model fields live on D3-branes, then $\alpha_{\mathrm{GUT}}=\tilde{g}_{\mathrm{s}}$, where the tilde refers to the value of the dilaton at the position of the Standard Model D3-brane; if in addition the dilaton is constant as in IIB orientifold models, then $g_{\mathrm{s}}=\tilde{g}_{\mathrm{s}}=\alpha_{\mathrm{GUT}}$. Unification in such models is necessarily nonstandard (see e.g. [23]), but generally $\alpha_{\mathrm{GUT}} \sim 0.05$. In F-theory compactifications on the other hand, the dilaton varies strongly over the compact space and there is no prediction for $g_{\mathrm{s}}$. Our calculations use perturbation theory in $g_{\mathrm{s}}$ and so are quantitatively valid only if $g_{\mathrm{s}}$ is somewhat less than one. Note that if $g_{\mathrm{s}}>1$ we may switch to a dual description in which $\mathrm{F}$ and $\mathrm{D}$ strings are interchanged and $g_{\mathrm{s}}^{\prime}=1 / g_{\mathrm{s}}<1$.

Furthermore, $p$ F-strings and $q$ D-strings can bind to form a $(p, q)$ string with tension

$$
\mu_{(p, q)}=\frac{\mu_{\mathrm{F}}}{g_{\mathrm{s}}} \sqrt{(p-C q)^{2} g_{\mathrm{s}}^{2}+q^{2}}
$$

We have now included the expectation value of the $\mathrm{RR}$ scalar $C$. This multiplet of strings was discovered through the $S L(2, Z)$ duality of the IIB string [24], and later explained in terms of FD bound states [25]. For relatively prime $p$ and $q$, the $(p, q)$ string has a nonzero binding tension with respect to any decomposition. For $p=n p^{\prime}$ and $q=n q^{\prime}$, the $(p, q)$ string is neutrally stable to splitting into $n\left(p^{\prime}, q^{\prime}\right)$ strings. Supersymmetry breaking will lead to a weak potential between them, but this is likely to be negligible for most purposes. The $n$ strings will in any case move on a common classical trajectory.

The integers $(p, q)$ can have either sign and are defined with respect to a specified orientation of the string; reversing the orientation sends $(p, q) \rightarrow(-p,-q)$. Three $(p, q)$ strings can meet provided

$$
p_{1}+p_{2}+p_{3}=q_{1}+q_{2}+q_{3}=0,
$$


where all strings are defined as pointing into the vertex. The vertex is essentially massless, so the angles at which the strings meet is fixed by the requirement that there be no force on the vertex. In its rest frame this implies that the strings lie in a plane, and that the angle between strings $i$ and $j$ is [26]

$$
\cos \hat{\theta}_{i j}=\frac{e_{i} \cdot e_{j}}{\left|e_{i}\right|\left|e_{j}\right|}, \quad e_{i}=\left(\left[p_{i}-C q_{i}\right] g_{\mathrm{s}}, q_{i}\right) .
$$

The string network evolves according to the Nambu-Goto action for each segment, with the constraint (2.4) where segments meet. This constraint can also be incorporated into the dynamics by assigning a small mass to the junction and allowing it to respond to the tensions of the attached strings.

The network can also change discontinuously when two strings collide. When $\left(p_{1}, q_{1}\right)$ and $\left(p_{2}, q_{2}\right)$ strings collide, they will pass through one another or reconnect in one of two ways as in figure $2 .^{5}$ If $\theta$ is less than the angle $\hat{\theta}_{12}$ defined in eq. (2.4), it

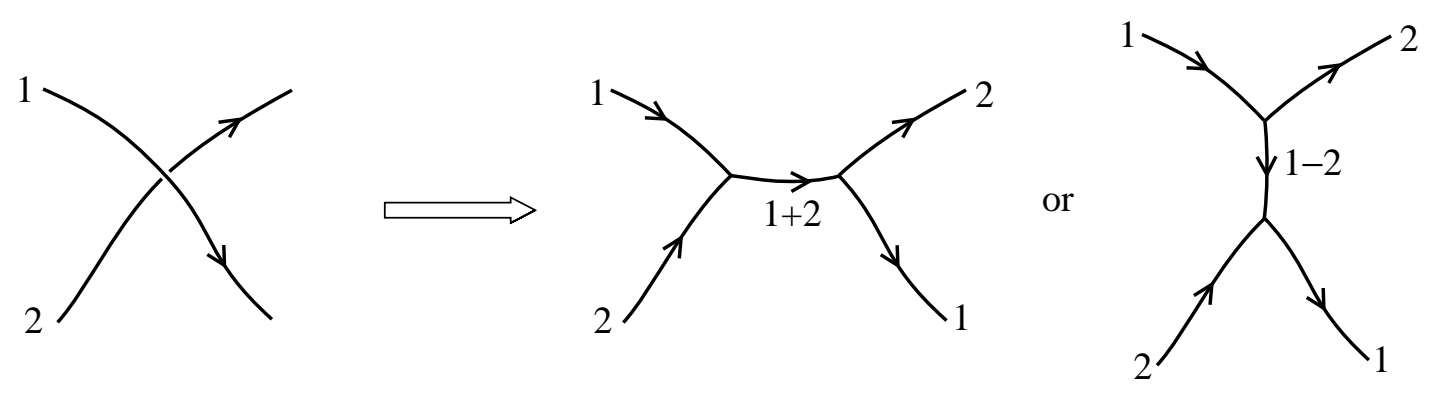

Figure 2: Possible reconnections of $\left(p_{1}, q_{1}\right)$ and $\left(p_{2}, q_{2}\right)$ strings; $i$ stands for $\left(p_{i}, q_{i}\right)$.

is energetically favorable to form a segment of $\left(p_{1}+p_{2}, q_{1}+q_{2}\right)$ string, and this is the presumed final state. If $\theta$ is greater than $\hat{\theta}_{12}$, the $\left(p_{1}-p_{2}, q_{1}-q_{2}\right)$ string is favored. The behavior of string networks will also depend on what happens when two string vertices meet, the inverse of the process shown in figure 2 . We will take this subject up in $\S 6$.

The $(p, q)$ strings are one-dimensional as seen either by a four-dimensional physicist or by a ten-dimensional physicist. Four-dimensional strings can also arise from higher-

\footnotetext{
${ }^{5}$ Another possibility would be that the two strings would stick together at a four-string vertex. Such higher vertices exist in some string models, where they arise from wrapped branes and are referred to as 'baryons' because of their role in gauge/string duality. However, they are rather massive and so unlikely to form in most cases.
} 
dimensional objects, $p$-branes ${ }^{6}$ in which $p-1$ dimensions are wrapped on part of the compact space and only one is extended in the visible directions. The IIB string has odd-dimensional D-branes, for example. Our results for D-strings (D1-branes) can be extended to these in a straightforward way. The IIB string also has an NS5-brane, which is a classical soliton like the ordinary cosmic string. When inflation arises from a D3/anti-D3 system, as for example in the models [6], only one-dimensional branes are produced [3].

The IIA theory has a fundamental string, even-dimensional D-branes and an NS5brane, and the same considerations apply. Similarly the type I theory has D1 and D5-branes (the fundamental type I string decays immediately by breakage on the spacefilling D9), the heterotic theory has an F-string and an NS5-brane, and M-theory has M2- and M5-branes. The heterotic theory also has fundamental gauge fields, and associated with these there can be electric and magnetic flux tubes which may be interesting cosmic strings [1]; the same applies to the low energy gauge theories that arise on branes. Indeed, it seems that these should be regarded, roughly speaking, as dual to the non-BPS strings identified in ref. [8]. In particular the magnetic flux tubes and D-strings both have large reconnection probabilities, while the electric flux tubes and F-strings have reconnection probabilities suppressed by $1 / N_{\mathrm{c}}^{2}$ and $g_{\mathrm{s}}^{2}$ respectively.

\section{$3 \quad \mathbf{F}-\mathbf{F}$ reconnection}

\subsection{Leading order}

We wish to calculate the probability for the process shown in figure 1. This was done in ref. [13] for the bosonic string; we review the method and extend it to the supersymmetric case.

Locally near the intersection, the process is two infinite straight strings going to two infinite bent strings. To make the process four-dimensional we compactify the six transverse dimensions on a six-torus of volume $V_{\perp}$. In order to use familiar vertex operator methods, we also wrap the long strings on a two-torus of lengths $l_{1,2}$ and angle $\theta$; at the end we take the two-torus volume to infinity. The resulting process, shown in figure 3 , is two unexcited winding strings going to an excited winding string with two kinks. The vertex operators for the two initial strings are much simpler than that for

\footnotetext{
${ }^{6}$ We use the standard terminology, but note that this is a different $p$ from the $(p, q)$ strings.
} 

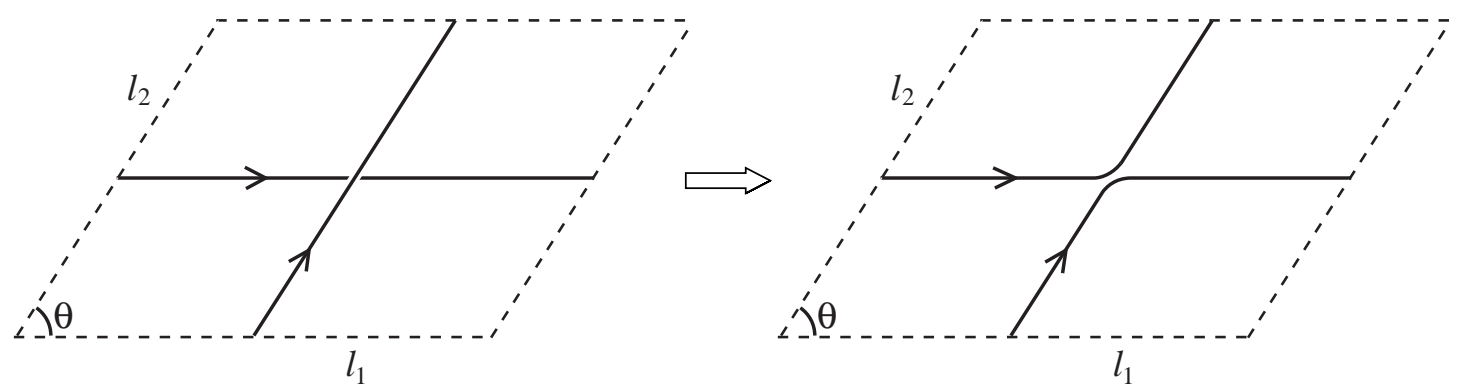

Figure 3: F-F reconnection with strings wound on a torus. This is a closed + closed $\rightarrow$ closed transition.

the final string. Fortunately, since we are interested in the total interaction probability, we can square and sum over intermediate states. By unitarity, this is related to the imaginary part of the tree level amplitude with four unexcited winding strings.

To simplify the supersymmetric calculation, we take the toroidal identifications to include a factor of $(-1)^{\mathbf{F}}$, where $\mathbf{F}$ is the spacetime fermion number. That is, we are treating both directions on the torus as 'temperature' directions. This gives the opposite of the usual GSO projection, so the ground states are scalars. Physically, adding a finite number of excitations to a long string cannot change the result.

The quantization conditions for the ground state strings of unit winding number are then

$$
p_{L}^{2}=p_{R}^{2}=\frac{2}{\alpha^{\prime}}, \quad p_{L / R}=p \pm \frac{L}{2 \pi \alpha^{\prime}} .
$$

The vertex operators in the $(-1,-1)$ and $(0,0)$ pictures are:

$$
\begin{aligned}
\mathcal{V}^{(-1,-1)} & =\frac{\kappa}{2 \pi \sqrt{V}}: e^{-\phi-\tilde{\phi}+i p_{L} \cdot X+i p_{R} \cdot \tilde{X}}:, \\
\mathcal{V}^{(0,0)} & =\frac{\kappa}{2 \pi \sqrt{V}} \frac{\alpha^{\prime}}{2}\left(\psi \cdot p_{L}\right)\left(\tilde{\psi} \cdot p_{R}\right): e^{i p_{L} \cdot X+i p_{R} \cdot \tilde{X}}: .
\end{aligned}
$$

Here $V=V_{\perp} l_{1} l_{2} \sin \theta$ is the eight-dimensional compactification volume, and the factor of $V^{-1 / 2}$ is from the zero modes.

The appropriate vertex operator correlator is

$$
\mathcal{A}=\left\langle\mathcal{V}_{1}^{(0,0)} \mathcal{V}_{2}^{(0,0)} \mathcal{V}_{3}^{(-1,-1)} \mathcal{V}_{4}^{(-1,-1)}\right\rangle
$$

where by the Riemann-Roch theorem there must be two vertex operators in the -1 picture, and the position of the first three operators are fixed to $z=0, z=1$ and 
$z \rightarrow \infty$ with $c$-ghosts. Also, because we use the optical theorem, we shall later set $p^{3}=-p^{1}$ and $p^{4}=-p^{2}$. Then

$$
\mathcal{A}=(2 \pi)^{2} \delta^{(2)}\left(\sum_{i} p_{i}\right) \frac{N_{\mathbb{S}^{2}} \kappa^{4}}{(2 \pi)^{4} V^{2}}\left(\frac{\alpha^{\prime}}{2} p_{L 1} \cdot p_{L 2}\right)^{2}\left|z_{4}\right|^{\alpha^{\prime} p_{L 1} \cdot p_{L 4}}\left|1-z_{4}\right|^{\alpha^{\prime} p_{L 2} \cdot p_{L 4}} .
$$

The normalization of the path integral is crucial, and is $N_{\mathbb{S}^{2}}=32 \pi^{3} V / \kappa^{2} \alpha^{\prime}$ [27]; the additional volume of compactification, $V$ comes from the zero-mode integrals. We have used the fact that in this calculation there is never momentum in the wound directions, so $p_{L i} \cdot p_{L j}=p_{R i} \cdot p_{R j}$. The positions of the first three operators are fixed; integrating over the fourth gives an invariant amplitude

$$
\mathcal{M}=-\frac{4 \kappa^{2}}{V \alpha^{\prime}} \frac{\Gamma\left(-\frac{\alpha^{\prime}}{4} s\right) \Gamma\left(-\frac{\alpha^{\prime}}{4} t\right) \Gamma\left(-\frac{\alpha^{\prime}}{4} u\right)}{\Gamma\left(1+\frac{\alpha^{\prime}}{4} s\right) \Gamma\left(1+\frac{\alpha^{\prime}}{4} t\right) \Gamma\left(1+\frac{\alpha^{\prime}}{4} u\right)},
$$

where $s, t$ and $u$ are the Mandelstam variables, constructed from either of $p_{L i}$ or $p_{R i}$.

We construct the angled F-string pair wrapped on the torus, with one string stationary and the other travelling toward it at velocity $v$, by setting

$$
\begin{aligned}
& p_{1}=\left[\left(\frac{l_{1}}{2 \pi \alpha^{\prime}}\right)^{2}-\frac{2}{\alpha^{\prime}}\right]^{\frac{1}{2}}(1,0,0,0, \mathbf{0}), \quad L_{1}=l_{1}(0,1,0,0, \mathbf{0}), \\
& p_{2}=\left[\left(\frac{l_{2}}{2 \pi \alpha^{\prime}}\right)^{2}-\frac{2}{\alpha^{\prime}}\right]^{\frac{1}{2}}\left[1-v^{2}\right]^{-\frac{1}{2}}(1,0,0, v, \mathbf{0}), \quad L_{2}=l_{2}(0, \cos \theta, \sin \theta, 0, \mathbf{0}) .
\end{aligned}
$$

For $l \gg \sqrt{\alpha^{\prime}}$ with fixed small $t$ (corresponding to momentum transfer in the transverse directions) we are in the Regge region and ${ }^{7}$

$$
\mathcal{M}=-\frac{\kappa^{2}}{V} \frac{s^{2}}{t}\left(\alpha^{\prime} s / 4\right)^{\alpha^{\prime} t / 2} e^{-i \pi \alpha^{\prime} t / 4} .
$$

The normalization of the $t=0$ pole agrees with graviton exchange calculated in an effective field theory, and the full form is determined by this normalization plus the Regge behavior. By an extension of this observation we will be able to obtain the general $\mathrm{F}-\mathrm{X}$ reconnection probability from field theory.

Inserting standard kinematic factors, the optical theorem gives

$$
\begin{aligned}
P & =\left.\frac{1}{4 E_{1} E_{2} v} 2 \operatorname{Im} \mathcal{M}\right|_{t=0} \\
& =\frac{\kappa^{2}}{\alpha^{\prime} \pi V_{\perp}} f(\theta, v), \quad f(\theta, v)=\frac{\left(1-\cos \theta \sqrt{1-v^{2}}\right)^{2}}{8 \sin \theta v \sqrt{1-v^{2}}} .
\end{aligned}
$$

\footnotetext{
${ }^{7}$ The imaginary part of the exact expression 3.5 lies at discrete poles; this discreteness arises from the introduction of the two-torus. In using Stirling's approximation we average these poles into a cut, as appropriate for the large- $l_{i}$ limit.
} 
Note that the factors of $l_{i}$ have cancelled out to give a finite $l_{i} \rightarrow \infty$ limit. The result is the same as for the bosonic string [13], as we could have anticipated from the remark below eq. (3.7). That the center of mass frame was used in ref. [13], so our velocity $v$ is related to the velocity $v^{\prime}$ there by $v=2 v^{\prime} /\left(1+v^{\prime 2}\right)$. Also, we have corrected an error in the sign of the $\cos \theta$ term; the numerator in $f(\theta, v)$ now goes to zero in the supersymmetric limit $\theta=v=0$.

In terms of the dimensionless IIB string coupling $g_{\mathrm{s}}$,

$$
P=\frac{g_{\mathrm{s}}^{2}(2 \pi)^{6} \alpha^{\prime 3}}{V_{\perp}} f(\theta, v)=g_{\mathrm{s}}^{2} \frac{V_{\min }}{V_{\perp}} f(\theta, v),
$$

where $V_{\min }=\left(4 \pi^{2} \alpha^{\prime}\right)^{3}$ is the minimum volume of a six-torus in the sense of $T$-duality. This is dimensionless, as it must be. If instead we leave $d>4$ dimensions noncompact, the strings can miss each other and the relevant quantity is a cross-section of dimension [length $]^{d-4}$. The difference is all in the zero modes, and the cross section is still given by eq. (3.9), with $V_{\perp}$ the volume of the $(10-d)$-torus but $V_{\min }$ unchanged.

\subsection{Higher corrections}

It would be interesting to consider higher corrections to our result. These can come from higher loops in string perturbation theory and from processes in which additional closed strings are emitted during the reconnection process. ${ }^{8}$ For example, at order $g_{\mathrm{s}}^{4}$, there will be the one-loop correction to the reconnection process and also reconnection with the emission of one small closed string. There is no reason to expect that such corrections are unusually large, but it would be useful to have some estimate of their magnitude.

To obtain the next order corrections will require some refinement of the simple unitary argument. The imaginary part of the torus amplitude, representing the total interaction probability at order $g_{\mathrm{s}}^{4}$, contains the above processes but also other nonreconnection processes. In particular, there is an $O\left(g_{\mathrm{s}}^{2}\right)$ amplitude for the strings to pass through one another but for some of their oscillators to become excited in the process, for example from the gravitational interaction between them, and this process actually dominates the interaction probability at $O\left(g_{\mathrm{s}}^{4}\right)$. Because each string produces

\footnotetext{
${ }^{8}$ Processes in which additional winding strings are created, or the winding pair are deflected, involve a length of string of order $l$ and so are exponentially suppressed in $l$. This is similar to the exponential suppression of high-energy fixed-angle scattering, with winding in place of momentum [28].
} 
a conic geometry, after they pass through one another they are no longer straight: each has a kink, its ends being misaligned by the deficit angle of order $O\left(g_{\mathrm{s}}^{4}\right)$. This kink can be described as a coherent state, an eigenstate of the mode operators $\alpha(k), \tilde{\alpha}(k)$ (these are the continuum version of the usual $\alpha_{n}, \tilde{\alpha}_{n}$, taking the limit of an infinite string). A linear kink in $X$ translates into $\alpha(k), \tilde{\alpha}(k) \propto 1 / k$. The norm of such a state is

$$
\int_{1 / l}^{\infty} \frac{d k}{k^{3}} \sim l^{2},
$$

diverging in the large-volume limit. This appears to agree with the string calculation: the $t \rightarrow 0$ behavior is given by the eikonal approximation [29], and in gravity each loop brings in an additional power of $s \sim l^{2}$.

It is likely that one can deal with this problem, and still take advantage of unitarity, by separating the contributions of different channels to the imaginary part of the one-loop amplitude. Obtaining a tractable form may be difficult; whereas the exponentially suppressed amplitudes are dominated by a saddle point in moduli space [30], the forward amplitude is not. One expects that the physics of the reconnection pro-

cess is local, and so the higher corrections will have a good $l \rightarrow \infty$ limit, unlike the IR-divergent process (3.10). It would be interesting to verify this.

\section{$4 \quad \mathbf{F}-(p, q)$ reconnection}

When an F-string crosses a D-string it can break, leaving its endpoints attached to the D-string. Taking both strings to be wound on a torus as in the previous case gives the process shown in figure 4. This is a closed-to-open transition for the Fstring. Following ref. [14] we again use the optical theorem, obtaining the the total interaction probability from the imaginary part of the amplitude for two closed string vertex operators on the disk,

$$
\mathcal{A}=\left\langle\mathcal{V}_{2}^{(0,0)} \mathcal{V}_{4}^{(-1,-1)}\right\rangle
$$

The vertex operators represent the winding F-string (and we have numbered them to match the previous section), while the D-string, which is stationary and oriented in the 1-direction, appears through the boundary conditions. The difference from ref. [14] is that we are considering the superstring rather than the bosonic string, and D1-brane boundary conditions rather than the fully Neumann D25-brane boundary conditions. 


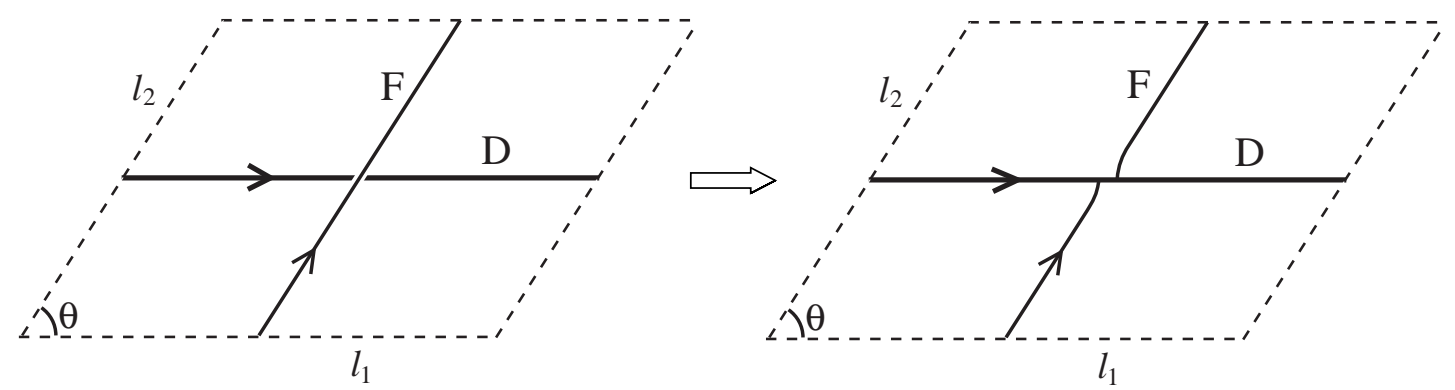

Figure 4: F-D reconnection with strings wound on a torus. This is a closed $\rightarrow$ open transition.

Since it is straightforward to do so, we consider the general $\mathrm{F}-(p, q)$ reconnection process. The $(p, q)$ string gives a boundary with a $q$-valued Chan-Paton factor, while the $p$ bound F-strings appear as constant $U(1)$ electric flux on the D-branes. ${ }^{9}$ The world-sheet CFT is free in such a background and the propagators are well known (see e.g. $[31,32])$,

$$
\begin{aligned}
& \left\langle X^{\mu}\left(z_{1}\right) X^{\nu}\left(z_{2}\right)\right\rangle=-\frac{\alpha^{\prime}}{2} \eta^{\mu \nu} \ln \left(z_{1}-z_{2}\right), \quad\left\langle\psi^{\mu}\left(z_{1}\right) \psi^{\nu}\left(z_{2}\right)\right\rangle=\frac{\eta^{\mu \nu}}{z_{1}-z_{2}} \\
& \left\langle X^{\mu}\left(z_{1}\right) \tilde{X}^{\nu}\left(\bar{z}_{2}\right)\right\rangle=-\frac{\alpha^{\prime}}{2} G^{\mu \nu} \ln \left(z_{1}-\bar{z}_{2}\right), \quad\left\langle\psi^{\mu}\left(z_{1}\right) \tilde{\psi}^{\nu}\left(\bar{z}_{2}\right)\right\rangle=\frac{G^{\mu \nu}}{z_{1}-\bar{z}_{2}} .
\end{aligned}
$$

Here

$$
G^{\mu \nu}=\left(\begin{array}{ccc}
-\frac{1+f^{2}}{1-f^{2}} & -\frac{2 f}{1-f^{2}} & 0 \\
\frac{2 f}{1-f^{2}} & \frac{1+f^{2}}{1-f^{2}} & 0 \\
0 & 0 & -\mathbb{1}
\end{array}\right)
$$

This is the open string metric in the $0-1$ plane, while the $-\mathbb{1}$ reflects the Dirichlet boundary condition in the other directions. The parameter $f$ is related to the electric flux and the number of bound F- and D-strings by

$$
f=2 \pi \alpha^{\prime} F_{01}, \quad p=\frac{q f}{g_{s} \sqrt{1-f^{2}}} .
$$

Again we keep a finite momentum transfer before taking the limit, so that $p_{4}$ is $-p_{2}$ with a scattered velocity $\boldsymbol{v}^{\prime}$ in the $3 \ldots 9$ directions. To leading order in $g_{\mathrm{s}}$ the D-string

\footnotetext{
${ }^{9}$ The bound state involves strongly coupled infrared dynamics for the $S U(q)$ degrees of freedom [25], but the process that we are considering takes place at the string scale and so is infrared-safe.
} 
does not recoil and $\left|\boldsymbol{v}^{\prime}\right|=v$. The invariants relevant to the calculation are then given in terms of two variables $\sigma$ and $t$ :

$$
\begin{aligned}
\frac{\alpha^{\prime}}{2} p_{2 L} \cdot G \cdot p_{2 R} & =\frac{\alpha^{\prime}}{2} p_{4 L} \cdot G \cdot p_{4 R} \equiv-\sigma \\
\frac{\alpha^{\prime}}{2} p_{2 L} \cdot G \cdot p_{4 R} & =\frac{\alpha^{\prime}}{2} p_{4 L} \cdot G \cdot p_{2 R} \equiv \sigma-\frac{\alpha^{\prime} t}{4} \\
\frac{\alpha^{\prime}}{2} p_{2 L} \cdot p_{4 L} & =\frac{\alpha^{\prime}}{2} p_{2 R} \cdot p_{4 R}=-1-\frac{\alpha^{\prime} t}{4} .
\end{aligned}
$$

We fix 3 of the 4 coordinates of the two vertex operators by $z_{2}=i$ and $z_{4}=i x$ with $x \in[0,1]$, and insert the corresponding $c$-ghosts. Evaluating the various factors in the expectation value gives

$$
\mathcal{M}=N_{\mathbb{D}^{2}} \frac{\kappa^{2} \sigma 2^{-2 \sigma}}{(2 \pi)^{2} V} \int_{0}^{1} d x(1-x)^{-1-\alpha^{\prime} t / 2}(1+x)^{1+2 \sigma+\alpha^{\prime} t / 2} x^{-1-\sigma} .
$$

Making a change of variables to $x=(1-\sqrt{y}) /(1+\sqrt{y})$ gives the standard representation of the beta function, and so [32]

$$
\begin{gathered}
\mathcal{M}=-N_{\mathbb{D}^{2}} \frac{\kappa^{2}}{(2 \pi)^{2} V} \frac{\Gamma\left(-\frac{\alpha^{\prime}}{4} t\right) \Gamma(1-\sigma)}{\Gamma\left(-\frac{\alpha^{\prime}}{4} t-\sigma\right)} \\
\stackrel{\text { Regge }}{\rightarrow}-N_{\mathbb{D}^{2}} \frac{\kappa^{2}}{(2 \pi)^{2} V} \frac{4}{\alpha^{\prime} t} \sigma^{1+\alpha^{\prime} t / 4} e^{-i \pi t \alpha^{\prime} / 4} .
\end{gathered}
$$

The normalization of the disc partition function with these boundary conditions is $N_{\mathbb{D}^{2}}=2 \pi^{2} l_{1} q \sqrt{1-f^{2}} / 2 \pi \alpha^{\prime} g_{s}$. This can be obtained from the standard disc partition function normalization, $2 \pi^{2} V_{9} \tau_{9}$, by $T$-duality, taking into account the Chan-Paton factors and the background fields. Note that each expression is $2 \pi^{2}$ times the total Born-Infeld action for the D-branes. One can check/verify this normalization by taking the extreme relativistic limit, where $\sigma \rightarrow 2 l_{2} /\left(1-f^{2}\right)\left(1-v^{2}\right) 2 \pi \alpha^{\prime}$. The $t=0$ pole is

$$
\mathcal{M} \rightarrow-\frac{\kappa^{2}}{V} \frac{2 l_{1} \tau_{p, q} l_{2}^{2}}{t\left(2 \pi \alpha^{\prime}\right)^{2}\left(1-v^{2}\right)} .
$$

This has the same normalization as the pole in eq. 3.7 upon replacing $s \rightarrow-2 p_{1} \cdot p_{2} \rightarrow$ $2 m_{p, q} E_{2}$; note that we are in the $(p, q)$ string rest frame. Also, there is a factor $1 / 2 m_{p, q}$ because we are implicitly using canonical rather than relativistic normalization for the $(p, q)$ string. 
Finally, the reconnection probability is

$$
\begin{aligned}
P & =\left.\frac{1}{2 E_{2} v} 2 \operatorname{Im} \mathcal{M}\right|_{t=0} \\
& =g_{\mathrm{s}}^{2} \frac{V_{\min }}{V_{\perp}} h_{p, q}(\theta, v), \\
h_{p, q}(\theta, v) & =\frac{q^{2} v^{2}+\left[g_{s} p-\cos \theta \sqrt{\left(1-v^{2}\right)\left(g_{s}^{2} p^{2}+q^{2}\right)}\right]^{2}}{8 \sin \theta v g_{s} \sqrt{\left(1-v^{2}\right)\left(g_{s}^{2} p^{2}+q^{2}\right)}}
\end{aligned}
$$

To confirm this, we have also obtained it by a very different route: T-dualing the 1direction and boosting to give $q$ stationary D0 branes interacting with F-string winding states in motion. Note that to leading order in perturbation theory, the $p$-dependence is important only if $p$ is of order $1 / g_{\mathrm{s}}$.

The disk calculation of the $\mathrm{F}-(p, q)$ probability (4.9) requires at least one D1-brane, and so is not valid for $(p, q)=(1,0)$; in the latter case we have instead the sphere amplitude of $\S 3$. Nevertheless the probability (4.9) reduces to the earlier result (3.9) in this case, and so (4.9) is universal in $p$ and $q$. This can be understood in a simple way. We have already noted that the amplitudes can be normalized by a gravity calculation of the $1 / t$ pole; taking this further, the full form of the $1 / t$ pole can be obtained from a supergravity calculation. The reconnection probability depends on the imaginary part as $t \rightarrow 0$. This imaginary part comes in both calculations from

$$
\operatorname{Im} e^{-i \pi \alpha^{\prime} t / 4}=-\sin \left(\pi \alpha^{\prime} t / 4\right) .
$$

The zero of the sine cancels the pole, giving a finite $t \rightarrow 0$ limit which is proportional to the supergravity amplitude.

We will not carry out the full supergravity calculation but we can check the $(p, q)$ dependence of some terms. The $\cos ^{2} \theta$ term, which comes only from graviton exchange, is proportional to the tension of the $(p, q)$ string. The $\cos \theta$ term, which comes only from $B_{\mu \nu}$ exchange, is proportional to the $\mathrm{F}$-string charge $p$. (The angle-independent term is more complicated because both the graviton and the dilaton exchanges contribute.) Also, at zero velocity the numerator vanishes when the angle between the strings is $\tan \theta=q / g_{s} p$, which is the angle at which the strings are mutually BPS and there is no long-range force between them [26].

We should emphasize that the imaginary part itself is not a supergravity effect. It is analytic in $t$ and so local in spacetime. Rather, the connection is that the total cross 
section is given by the leading Regge trajectory at $t=0$, which is the supergravity amplitude. The factor (4.10), which arises from the continuation of Regge amplitude $(-s)^{\alpha^{\prime} t / 4}$ from Euclidean to Lorentzian momenta, provides the connection between the pole and the imaginary part.

Is this connection completely universal, so that we can immediately write down the general $(p, q)-\left(p^{\prime}, q^{\prime}\right)$ result? In the disk and sphere calculations, the factor (4.10) has a common world-sheet origin. In the Regge region, the in and out vertex operators $\mathcal{V}_{2}$ and $\mathcal{V}_{4}$ are at small separation, and give a universal Regge form which is the same whether the rest of the world-sheet is a sphere with other vertex operators or a disk. One might have expected this Regge behavior to be completely universal, but in the general $(p, q)-\left(p^{\prime}, q^{\prime}\right)$ interaction that we are about to consider, the world-sheet origin of the imaginary part is different, and the final result shows no sign of universality.

\section{$5 \quad(p, q)-\left(p^{\prime}, q^{\prime}\right)$ reconnection}

\subsection{Pair production}

When both strings have D1 charge, the leading interaction between them comes from annular world-sheets with one boundary on each. This calculation was done for parallel $\mathrm{D} p$-branes by Bachas [15]; it has been extended and applied many times since, particularly in the small-velocity expansion relevant to matrix theory.

We start with the case of two $(p, q)=(0,1)$ D-strings, with one aligned at an angle $\theta$ and travelling at speed $v \equiv \tanh (\pi \epsilon)$ relative to the other, and with impact parameter $y$. It is straightforward to extend the results of ref. [15] to this case [27,33-35],

$$
\begin{aligned}
\mathcal{M}(y)=-\frac{i}{2} \int_{0}^{\infty} \frac{d t}{t} e^{-t y^{2} / 2 \pi \alpha^{\prime}} & {\left[\eta^{6}(i t) \Theta_{1}\left(i \frac{\theta t}{\pi} \mid i t\right) \Theta_{1}(\epsilon t \mid i t)\right]^{-1} } \\
\times & \left\{\sum_{k=2}^{4}(-1)^{k-1} \Theta_{k}(0 \mid i t)^{2} \Theta_{k}\left(i \frac{\theta t}{\pi} \mid i t\right) \Theta_{k}(\epsilon t \mid i t)\right\} .
\end{aligned}
$$

The total inelastic probability $P_{\mathrm{pp}}$ can be put in a simple universal form due to Schwinger [36]. This corresponds to production of at least one pair of stretched strings. It is not the same as the reconnection probability, as we will explain in $§ 5.5$. Summing over disconnected annuli gives

$$
1-P_{\mathrm{pp}}(y)=\left|e^{i \mathcal{M}(y)}\right|^{2}=e^{-2 \operatorname{Im} \mathcal{M}(y)} .
$$


The imaginary part arises from the poles of $\Theta_{1}(\epsilon t \mid i t)^{-1}$ on the real $t$-axis, at $t=n / \epsilon$. These are all traversed on the same side [15]:

$$
\operatorname{Im} \mathcal{M}(y)=\frac{1}{2} \sum_{n=1}^{\infty} \frac{1}{n}\left[(-1)^{n+1} Z_{B}(n / \epsilon)+Z_{F}(n / \epsilon)\right],
$$

where partition functions are

$$
\begin{aligned}
& Z_{B}(t) \equiv \sum_{\text {bosons } i} e^{-2 \pi \alpha^{\prime} t m_{i}^{2}}=e^{-t y^{2} / 2 \pi \alpha^{\prime}} \frac{\Theta_{3}(0 \mid i t)^{3} \Theta_{3}\left(i \frac{\theta t}{\pi} \mid i t\right)-\Theta_{4}(0 \mid i t)^{3} \Theta_{4}\left(i \frac{\theta t}{\pi} \mid i t\right)}{2 \eta^{9}(i t) i \Theta_{1}\left(i \frac{\theta t}{\pi} \mid i t\right)} \\
& Z_{F}(t) \equiv \sum_{\text {fermions } j} e^{-2 \pi \alpha^{\prime} t m_{j}^{2}}=e^{-t y^{2} / 2 \pi \alpha^{\prime}} \frac{\Theta_{2}(0 \mid i t)^{3} \Theta_{2}\left(i \frac{\theta t}{\pi} \mid i t\right)}{2 \eta^{9}(i t) i \Theta_{1}\left(i \frac{\theta t}{\pi} \mid i t\right)}
\end{aligned}
$$

The residues sum up to give

$$
1-P_{\mathrm{pp}}(y)=\prod_{\text {bosons } i}\left(1+x_{i}\right)^{-1} \prod_{\text {fermions } j}\left(1-x_{j}\right)
$$

where $x=e^{-2 \pi \alpha^{\prime} m^{2} / \epsilon}$ and $m$ is the mass of the given stretched string state at minimum separation.

\subsection{Small velocity limit}

To get some understanding of this result, consider the limit $\epsilon \ll 1$. Then

$$
x=e^{2 \pi \alpha^{\prime} m^{2} / \epsilon} \rightarrow\left\{\begin{array}{cc}
0, & m^{2}>0 \\
\infty, & m^{2}<0
\end{array}\right.
$$

Thus $P_{\mathrm{pp}}(y)=1$ if there is a tachyon in the spectrum, and $P_{\mathrm{pp}}(y)=0$ otherwise. This is the well-known fact that the annulus amplitude between non-BPS configurations of branes diverges at the critical impact parameter where a tachyon first appears [37]. At larger impact parameters, the small-velocity process is adiabatic and elastic. At smaller impact parameters, the tachyonic instability proceeds when the critical separation is reached.

The lightest string state in the present case is a boson with

$$
m^{2}=\frac{y^{2}}{\left(2 \pi \alpha^{\prime}\right)^{2}}-\frac{\theta}{2 \pi \alpha^{\prime}} .
$$


This is tachyonic for $y^{2}<2 \pi \alpha^{\prime} \theta$ [34]. For strings at angles there is an obvious final state for the tachyonic decay, namely the reconnected strings [38]; for some detailed studies of this process see refs. [39].

Note that the result is independent of the compactification volume, because the stretched strings are confined to the region near the intersection and have no zero modes. Also, the D-strings are treated as having definite classical trajectories. In the noncompact case this translates into a classical black-sphere cross section

$$
\sigma=\int d^{6} \boldsymbol{y} P_{\mathrm{pp}}(y)=\left(2 \pi^{2} \alpha^{\prime} \theta\right)^{3}
$$

For toroidal compactification, taking the D-strings to have a constant wavefunction in the compact directions gives

$$
P_{\mathrm{pp}}=\frac{\left(2 \pi^{2} \alpha^{\prime} \theta\right)^{3}}{V_{\perp}},
$$

where we assume that the $T^{6}$ is large enough to contain the black sphere without overlap.

We can now see that the supergravity argument does not extend to this case. It would give the same as the small-velocity limit of the F-F probability, except for an additional factor of $g_{\mathrm{s}}^{2}$ from the greater tension of the D-string:

$$
P_{\mathrm{pp}} \stackrel{?}{=} \frac{\left(2 \pi^{2} \alpha^{\prime}\right)^{3}}{V_{\perp}} \frac{(1-\cos \theta)^{2}}{v \sin \theta} .
$$

In spite of the similarity of these expressions, they definitely differ by a factor of $v$, and there seems to be no way to relate them. At the world-sheet level they have very different origins. The Regge region for the F-string processes comes from small $z_{24}$, corresponding to a long cylinder in the $t$-channel. The equivalent region for the annulus parameter $t$ (not to be confused with the Mandelstam $t$ ) is $t \ll 1$. On the other hand, for small $\epsilon$ we see that the poles (5.3) move to $t \gg 1$.

Of course, at $g_{\mathrm{s}}=1 \mathrm{~F}$-strings and D-strings are identical under duality and so the reconnection probabilities should become equal. This is not evident in the smallvelocity limit, where the $\mathrm{D}-\mathrm{D}$ interaction (5.9) approaches a constant while the $\mathrm{F}-\mathrm{F}$ result (3.8) diverges as $1 / v$. Higher order effects must cut the latter off; perhaps some simple unitarization along the lines of the eikonal approximation can be used to estimate this. 


\subsection{Ultrarelativistic velocities}

For $\epsilon \gg 1$, the poles in $t$ move to small values and so all string modes are significant in the sum (5.3). The asymptotics of the partition functions are given by a modular transformation,

$$
Z_{B}(t) \sim Z_{F}(t) \simeq \frac{t^{3}}{4 \sin \theta} e^{\pi / t}+O(1) .
$$

The $n=1$ term in the sum (5.3) dominates, giving

$$
\begin{aligned}
2 \operatorname{Im} \mathcal{M}(y) & \simeq \frac{1}{2 \epsilon^{3} \sin \theta} e^{\pi \epsilon-t y^{2} / 2 \pi \alpha^{\prime}} \\
& \simeq K e^{-t y^{2} / 2 \pi \alpha^{\prime}}, \quad K=\frac{8 \pi^{3}}{\sin \theta \sqrt{1-v^{2}}\left[-\ln \left(1-v^{2}\right)\right]^{3}} .
\end{aligned}
$$

We can now carry out the integral (5.8), using the fact that $K$ is large:

$$
\begin{aligned}
\sigma & =\pi^{3} \int_{0}^{\infty} d y y^{5}\left(1-e^{-2 \operatorname{Im} \mathcal{M}(y)}\right) \\
& \simeq \frac{\left(2 \pi^{2} \alpha^{\prime}\right)^{3}}{6}(\ln K)^{3} \\
& \simeq \frac{\left(2 \pi^{2} \alpha^{\prime}\right)^{3}}{48}\left[-\ln \left(1-v^{2}\right)\right]^{3} .
\end{aligned}
$$

As noted in ref. [15], this is similar to the hard scattering of F-strings.

\subsection{Cosmic collisions}

In cosmic string networks, the velocities are moderately relativistic, so that a typical string collision will have $v \sim 0.7$ or $\epsilon \sim 0.3[10] .{ }^{10}$ Surprisingly, this is not so different from the small velocity limit, in that only the lightest open strings are produced. An excited string with $m^{2}=1 / \alpha^{\prime}$ has

$$
x=e^{-2 \pi \alpha^{\prime} m^{2} / \epsilon} \lesssim 10^{-9} .
$$

When $x$ is small, it is the same as the probability to create a pair of strings in the given state, which is therefore negligible. For even higher states this probability decreases faster than the density of states increases, so only unexcited strings are produced with

\footnotetext{
${ }^{10}$ Of course, in an evolving network there will be a distribution of collision parameters.
} 
any probability. Thus we can restrict the partition functions (5.4) to those states whose mass goes to zero with $\theta$ :

$$
\begin{aligned}
& Z_{B}(t) \simeq e^{-t y^{2} / 2 \pi \alpha^{\prime}} \frac{6+2 \cosh 2 \theta t}{2 \sinh \theta t}=e^{-t y^{2} / 2 \pi \alpha^{\prime}}\left[e^{\theta t}+7 e^{-\theta t}+\ldots\right], \\
& Z_{F}(t) \simeq e^{-t y^{2} / 2 \pi \alpha^{\prime}} \frac{8 \cosh \theta t}{2 \sinh \theta t}=e^{-t y^{2} / 2 \pi \alpha^{\prime}}\left[4+8 e^{-2 \theta t}+\ldots\right] .
\end{aligned}
$$

As we will explain in $\S 7$, the mean value of $y^{2}$ in a realistic situation will be of order $g_{\mathrm{s}}$, and so we set it to zero in the spirit of this perturbative calculation. In $\S 7$ we will estimate corrections to this approximation, and we will find that they are likely to be quite substantial. Nevertheless an understanding of the case $y^{2}=0$ is instructive. A state contributing $e^{-k \theta}$ in $Z_{B, F}$ then has

$$
x \simeq e^{-k \theta / \epsilon},
$$

and so

$$
1-P_{\mathrm{pp}}(0)=\left(1+e^{\theta / \epsilon}\right)^{-1}(1-1)^{4}\left(1+e^{-\theta / \epsilon}\right)^{-7}\left(1-e^{-2 \theta / \epsilon}\right)^{8} \cdots .
$$

For angles of order one, $e^{-\theta / \epsilon} \lesssim 0.04$ and the first two terms in the infinite product dominate:

$$
1-P_{\mathrm{pp}}(0) \simeq\left(1+e^{\theta / \epsilon}\right)^{-1}(1-1)^{4} \lesssim 0.04 \times 0^{4} .
$$

That is, the production probability is at least 0.96 for a pair of the lightest bosonic strings, and exactly 1 for a pair of each of the four lightest fermionic strings. The reason for the exact zero in $1-P_{\mathrm{pp}}(0)$ is that the mass of the lowest fermionic states passes through zero and so there is a level crossing: the empty in-state becomes the filled out-state.

For smaller angles the tachyonic term becomes smaller but the higher terms in the series rapidly begin to contribute. Numerically, the probability to produce a pair, aside from the four fermionic zero mode pairs, reaches a minimum around 0.95 for $\theta / \epsilon \sim 2$. In realistic situations, the fermionic zero modes will be lifted somewhat by couplings to supersymmetry-breaking fluxes. However, the net $P_{\mathrm{pp}}(0)$ will in most cases remain close to one, as long as the effective impact parameter $y$ does not become too large.

\subsection{Reconnection}

For $g_{\mathrm{s}} \ll 1$, the D-strings are much heavier than the F-strings. Production of a single pair of F-strings will not cause the D-strings to reconnect. Rather, the D-strings will 
pass through one another and separate, stretching the F-strings as they do so. Thinking of the D-strings as open string solitons, one would expect that of order $g_{\mathrm{s}}^{-1} \mathrm{~F}$-strings must be produced in order to produce a substantial change in the state of the D-strings.

We can make this estimate precise as follows. After the collision the system is as shown in figure 5. For $N$ F-string pairs, balance of forces implies that the angle

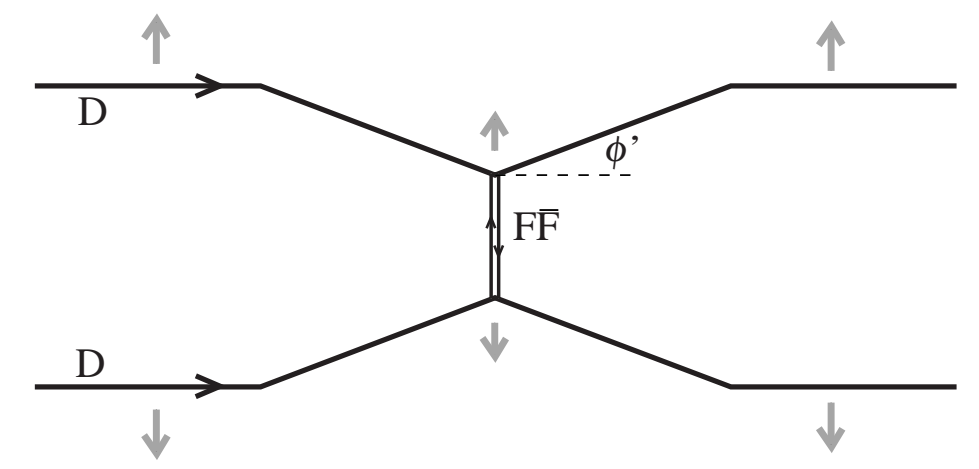

Figure 5: D-strings after a collision that produces open F-string pairs. The upper D-string is rotated by an angle $\theta$ relative to the lower, around the axis defined by the F-strings. The angle $\phi^{\prime}$ is the angle $\phi$ introduced in the text, boosted by the velocity of the vertex.

between the F-strings and D-strings in the rest frame of the junction is $\frac{1}{2} \pi+\phi$, where $\sin \phi=N g_{\mathrm{s}}$. The effect of the collision can only travel with the speed of light, so on each D-string there are two kinks traveling away from the point of the collision. This picture only makes sense if $u>\tan \phi$, where $u=\tanh (\pi \epsilon / 2)$ is the speed of each D-string in the center-of-mass frame. If this is not satisfied the two junctions do not separate and the D-strings remain in contact (the vertices are at rest at the crossover velocity, so the boost of the junction angle does not enter). For $u>\tan \phi$, where we have the situation in figure 5, the ends of the open strings are forced to remain localized and we expect that ends of opposite orientation will rapidly find one another and annihilate. The two D-strings thus disconnect and continue onward, with some excitation. For $u<\tan \phi$, we would expect that the D-strings will instead roll down to the reconnected configuration. Thus the condition for reconnection is $u<\tan \phi$, or equivalently

$$
N>\frac{1}{g_{\mathrm{s}}} \sinh (\pi \epsilon / 2)
$$


In order to apply this, we need to refine the earlier calculation, which just determined the probability that $N \geq 1$. For the case (5.18) this is simple. The tachyon pairs are produced in a squeezed state. For a squeezed state of a single oscillator, if the probability of producing at least one pair is $p$, then the probability of producing at least $k$ pairs is just $p^{k}$. Here, $p \simeq 1-e^{-\theta / \epsilon}$ and so $p^{k} \simeq \exp \left(-k e^{-\theta / \epsilon}\right)$. Counting the four fermionic pairs, the probability that the reconnection condition (5.19) is satisfied is

$$
P=\exp \left(\left[4-g_{\mathrm{s}}^{-1} \sinh (\pi \epsilon / 2)\right] e^{-\theta / \epsilon}\right) .
$$

We see the somewhat surprising result that $P$ decreases as $g_{\mathrm{s}} \rightarrow 0$, because the reconnection condition (5.19) becomes more stringent while the probability of producing a given number of pairs is constant. In fact, it falls as $e^{-O\left(1 / g_{\mathrm{s}}\right)}$ and so is nonperturbative, even though it was deduced from a perturbative calculation. At asymptotically small couplings D-D reconnection is much less likely than $\mathrm{F}-\mathrm{F} .{ }^{11}$

This asymptotic suppression of $P$ does not set in until below the GUT value $g_{\mathrm{s}} \sim$ 0.05. For $g_{\mathrm{s}} \sim 0.05$ and $\epsilon \sim 0.3$, we have $P \sim \exp \left(-6 e^{-\theta / 0.3}\right)$. At $\theta \gtrsim 1, P$ is at least 0.8 ; for $\theta \sim 0.6$ it falls to around 0.5 and then begins to rise again due to the higher states in the expansion (5.15). Thus for this choice of parameters there is a range of small angles where D-strings will sometimes pass through one another, but this will have likely have a small effect on the network behavior.

\subsection{General $\left(p_{1}, q_{1}\right)-\left(p_{2}, q_{2}\right)$ interaction}

In the general case we have $q_{1} q_{2}$ Chan-Paton states for the open strings, and an electric flux (4.4) on each D-string from the dissolved F-strings. The boundary conditions can be written as [31]

$$
\begin{aligned}
& A^{\mu}{ }_{\nu} X^{\nu \prime}(0)=B^{\mu}{ }_{\nu} \dot{X}^{\nu}(0), \\
& C^{\mu}{ }_{\nu} X^{\nu \prime}(\pi)=D^{\mu}{ }_{\nu} \dot{X}^{\nu}(\pi) .
\end{aligned}
$$

Here

$$
A=\left[\begin{array}{llll}
1 & 0 & 0 & 0 \\
0 & 1 & 0 & 0 \\
0 & 0 & 0 & 0 \\
0 & 0 & 0 & 0
\end{array}\right], \quad B=B_{1}, \quad C=A R, \quad D=B_{2} R
$$

\footnotetext{
${ }^{11}$ When $g_{\mathrm{s}}, v$, and $\theta$ all go to zero, the result depends on their ratio.
} 
defined in terms of

$$
B_{i}=\left[\begin{array}{cccc}
0 & f_{i} & 0 & 0 \\
f_{i} & 0 & 0 & 0 \\
0 & 0 & 1 & 0 \\
0 & 0 & 0 & 1
\end{array}\right], \quad R=\left[\begin{array}{cccc}
\cosh \pi \epsilon & 0 & 0 & \sinh \pi \epsilon \\
0 & \cos \theta & \sin \theta & 0 \\
0 & -\sin \theta & \cos \theta & 0 \\
\sinh \pi \epsilon & 0 & 0 & \cosh \pi \epsilon
\end{array}\right] .
$$

Inserting a general linear combination of $e^{i \omega(\tau+\sigma)}$ and $e^{i \omega(\tau-\sigma)}$, one finds that $e^{2 \pi i \omega}$ must be an eigenvalue of

$$
\Lambda=R^{-1}\left(A-B_{2}\right)^{-1}\left(A+B_{2}\right) R\left(A+B_{1}\right)^{-1}\left(A-B_{1}\right) .
$$

Noting that

$$
\left(A+B_{i}\right)^{-1}\left(A-B_{i}\right)=\left[\begin{array}{cccc}
\cosh 2 \xi_{i} & -\sinh 2 \xi_{i} & 0 & 0 \\
-\sinh 2 \xi_{i} & \cosh 2 \xi_{i} & 0 & 0 \\
0 & 0 & -1 & 0 \\
0 & 0 & 0 & -1
\end{array}\right] \in S O(1,3)
$$

where $f_{i}=\tanh \xi_{i}$, it follows that $\Lambda \in S O(1,3)$. Any Lorentz transformation is conjugate to a boost times a commuting rotation, and so the eigenvalues take the same form as in the $\mathrm{D}-\mathrm{D}$ case

$$
\omega= \pm \tilde{\theta} / \pi, \pm i \tilde{\epsilon}
$$

in terms of an effective rotation angle $\tilde{\theta}$ and an effective rapidity $\pi \tilde{\epsilon}$. For example, we can put these in the form (obtained using the $S L(2, C)$ representation)

$$
\cosh (\pi \tilde{\epsilon}+i \tilde{\theta})=\cosh \xi_{1} \cosh \xi_{2} \cosh (\pi \epsilon+i \theta)-\sinh \xi_{1} \sinh \xi_{2}
$$

whose real and imaginary parts determine $\tilde{\theta}$ and $\pi \tilde{\epsilon}$. The full form is rather messy. One simple special case is a perpendicular $(p, q)-\mathrm{D}$ collision, $\theta=\pi / 2, f_{2}=0$, where $\tilde{\theta}=\pi / 2$ and $\sinh \pi \tilde{\epsilon}=\cosh \xi_{1} \sinh \pi \epsilon$.

The discussion of $\mathrm{D}-\mathrm{D}$ reconnection then extends directly to the general case. Note that $(1-P)$ is raised to the power $q_{1} q_{2}$ due to the Chan-Paton degeneracy. The general conclusion, that reconnection almost always occurs unless $g_{\mathrm{s}}$ is very small, continues to hold.

There is one special circumstance that we should note. Although they do not bind, $n$ fundamental strings can move together as though they were an $(n, 0)$ string. If these collide with another string, then in perturbation theory each will interact 
independently. On the other hand, if we have $m$ coincident D-strings (or other $(p, q)$ strings with $q \neq 0)$, and they cross a $\left(p^{\prime}, q^{\prime}\right)$ string with $q^{\prime} \neq 0$, then the rule of thumb is that the tachyon decay will almost always take them to a new lower energy configuration, with a segment of $\left(p^{\prime}, q^{\prime} \pm m\right)$ string, the sign depending on the angle.

\section{$6 \quad$ Vertex interactions}

As the string network evolves, pairs of trilinear vertices will collide as in figure 6 , and we need to determine the subsequent evolution. In the figure, we begin with the

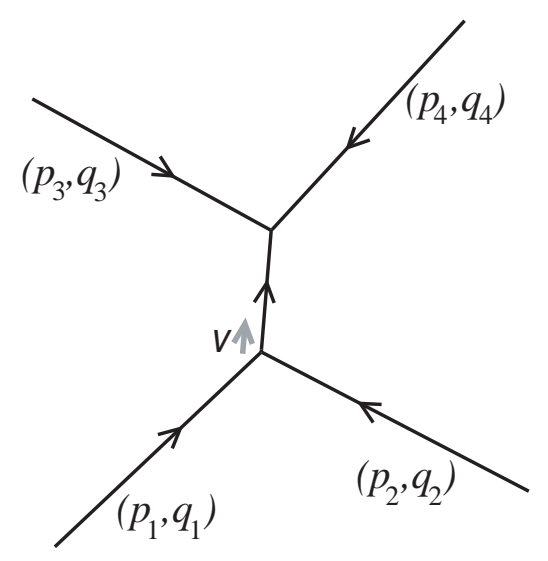

Figure 6: Vertex collision: the lower vertex is moving toward the other with speed $v$.

supersymmetric configuration in which string $i$ is in the direction $\left(p_{i} g_{\mathrm{s}}, q_{i}\right)$, and define a general configuration by rotating strings 3 and 4 by an angle $\psi$ around the string segment. The simplest case is two F-strings ending on a $(p, q)$ string, so that

$$
\left(p_{i}, q_{i}\right)=(p, q),(-1,0),(1,0),(-p,-q), \quad i=1,2,3,4 .
$$

The two F-strings have the same orientation, so the endpoints can annihilate and the F-string disconnect from the $(p, q)$ string.

We can obtain the probability for this by the same general strategy as for the previous F-string processes. To set up the macroscopic open string states, we introduce two spectator strings on which the other ends of the F-strings are fixed. This is shown in figure 7 ; note that to lowest order the $(p, q)$ strings do not bend when a single F-string attaches. These are at separation $R$ in the 2-3 plane, and we take $R \rightarrow \infty$ 
at the end of the calculation to remove the spectator strings and make the F-strings macroscopic. As before we take the opposite of the usual GSO projection so as to get the simpler scalar ground state; this is equivalent to taking the spectators to be $(p,-q)$ strings. We again make use of the optical theorem to obtain the contribution from all

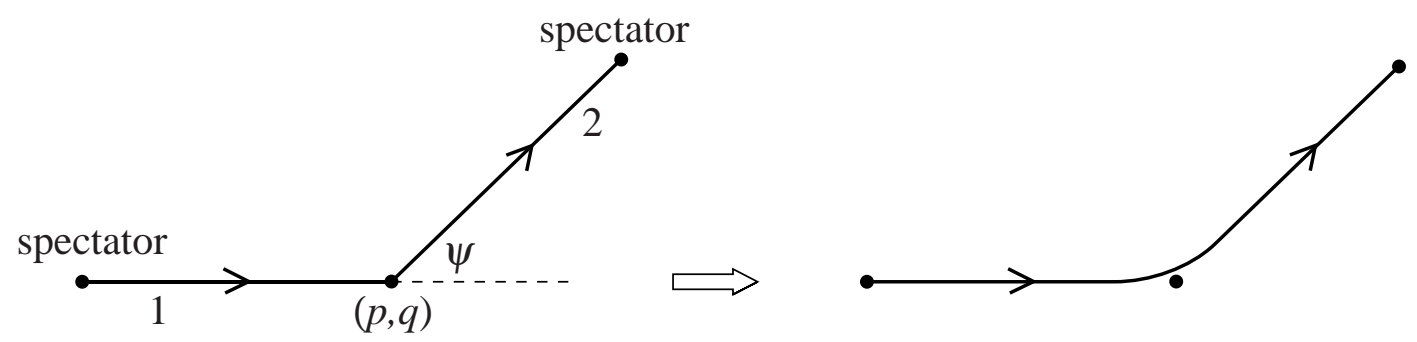

Figure 7: Disconnection process as seen along the axis of the $(p, q)$ string. With two spectator $(p, q)$ strings it becomes an open+open $\rightarrow$ open amplitude.

final states.

The disconnection process is characterized by the relative velocity of the endpoints and the relative angle $\psi$ of the two strings (figure 6 ). The open string vertex operators for the strings stretched between the branes are

$$
\begin{aligned}
\mathcal{V}^{(-1)} & =\lambda g_{\mathrm{o}}: e^{-\phi} e^{i p_{L} \cdot X+i p_{R} \cdot \tilde{X}}: \\
\mathcal{V}^{(0)} & =\lambda g_{\mathrm{o}} \sqrt{2 \alpha^{\prime}}(\psi \cdot p): e^{i p_{L} \cdot X+i p_{R} \cdot \tilde{X}}: .
\end{aligned}
$$

We have added adjoint $U(q)$ Chan-Paton factors $\lambda$ to the F-string vertex operators. Note also that there are no factors of $V_{\perp}$ since the open string wavefunctions are localized in the extra dimensions by the D-strings.

We align the $(p, q)$-string along the 1-direction, place the first F-string at rest aligned along the 2-direction, and the second with velocity along the $(p, q)$-string and aligned at an angle $\psi$ in the $2-3$ plane. The momenta are therefore

$$
\begin{aligned}
& p_{1 L, R}=\left[\left(\frac{R}{2 \pi \alpha^{\prime}}\right)^{2}-\frac{1}{2 \alpha^{\prime}}\right]^{\frac{1}{2}}\left[1-f^{2}\right]^{\frac{1}{2}}(1,0,0,0, \mathbf{0}) \pm \frac{R}{2 \pi \alpha^{\prime}}(0,0,1,0, \mathbf{0}), \\
& p_{2 L, R}=\left[\left(\frac{R}{2 \pi \alpha^{\prime}}\right)^{2}-\frac{1}{2 \alpha^{\prime}}\right]^{\frac{1}{2}}\left[\frac{1-f^{2}}{1-v^{2}}\right]^{\frac{1}{2}}(1, v, 0,0, \mathbf{0}) \pm \frac{R}{2 \pi \alpha^{\prime}}(0,0, \cos \psi, \sin \psi, \mathbf{0}) .
\end{aligned}
$$

We have renumbered relative to figure 6: the initial and final F-strings are now 1,2 and 3,4 respectively. The vertex operator for the stationary string can be obtained, 
for example, by $T$-duality along the 1-direction, and then the other is obtained by a boost. Using the contractions (4.2), each pair of vertex operators leads to a factor of $e^{2 \alpha^{\prime} p_{i} * p_{j}}$, where

$$
p_{i} * p_{j}=\frac{1}{4}\left(p_{i L} \cdot p_{j L}+p_{i R} \cdot p_{j R}+p_{i L} \cdot G \cdot p_{j R}+p_{j L} \cdot G \cdot p_{i R}\right) .
$$

One can check the mass shell condition $p_{i} * p_{i}=2 / \alpha^{\prime}$.

The amplitude is then

$$
\mathcal{M}=-\tilde{N}_{\mathbb{D}^{2}} g_{\mathrm{o}}^{4} \operatorname{Tr}\left(\lambda^{1} \lambda^{2} \lambda^{2 \dagger} \lambda^{1 \dagger}\right) \frac{\Gamma\left(-\alpha^{\prime} s\right) \Gamma\left(-\alpha^{\prime} t\right)}{\Gamma\left(1+\alpha^{\prime} u\right)},
$$

where the Mandelstam variables are defined by $s=-\left(p_{1}+p_{2}\right) *\left(p_{1}+p_{2}\right)$ and so on. The Chan-Paton trace is simply 1 (in all other channels it vanishes). The path integral is

normalized as in $\S 4, \tilde{N}_{\mathbb{D}^{2}}=\pi \sqrt{1-f^{2}} / \alpha^{\prime} g_{s}$ (we are now treating the 1-direction with continuum normalization, and separating out the explicit Chan-Paton trace), while $\tilde{N}_{\mathbb{D}^{2}} g_{\mathrm{o}}^{2}=1 / \alpha^{\prime}$ holds in general. Using these normalizations and taking the imaginary part as in earlier calculations,

$$
\operatorname{Im} \mathcal{M}=\frac{g_{\mathrm{s}} s}{\sqrt{1-f^{2}}}
$$

With the usual kinematic factors, the disconnection probability is

$$
P=\frac{2 \operatorname{Im} \mathcal{M}}{2 E_{1} 2 E_{2} v}=\frac{g_{\mathrm{s}}}{v} \frac{1-\sqrt{1-v^{2}} \cos \psi}{\left(1-f^{2}\right)^{3 / 2}} .
$$

When three or four of the strings carry D-string charge, there is no simple CFT description of the system. However, we can resort to the rule of thumb that in this situation the open string tachyons will almost always take the strings to their lowest energy state. We must still determine which of strings 3 and 4 string 1 will join onto. In fact, the supersymmetric configuration shown is neutrally stable in both directions, but any nonzero $\psi$ increases $\theta_{13}$ and decreases $\theta_{14}$ so that the latter reconnection is favored. In particular, when $\left(p_{1}, q_{1}\right)=-\left(p_{4}, q_{4}\right)$, the strings disconnect.

\section{Compactification effects}

The string reconnection probabilities (3.9, 4.9, 5.9) all depend on the compactification volume as $V_{\min } / V_{\perp}$, reflecting the fact that the strings have to come roughly within 
a string radius in order to interact $[4,5,7]$. It is therefore essential to determine the effective value of $V_{\perp}$.

Naively it would seem that one could obtain very small values of $P$ in models with large compact dimensions. However, from the point of view of the world-sheet field theory, the position of the string in the compact dimensions is a scalar field, which is not protected by any symmetry. One therefore expects that at some scale this modulus will be fixed, like the compactification moduli. That is, there is an effective potential which localizes the string. Moreover, the behavior of scalar fields in $1+1$ dimensions implies that the effective volume over which the string wavefunction spreads depends only logarithmically on the mass scale of the moduli - as the cube of the logarithm, to be precise [8]. As a result, $P$ can be suppressed somewhat, but not by many orders of magnitude.

\subsection{Generalities}

We obtain the effective action for a $(p, q)$ string moving in a general warped string metric

$$
d s^{2}=H^{-1 / 2}(Y) \eta_{\mu \nu} d X^{\mu} d X^{\nu}+H^{1 / 2}(Y) g_{i j}(Y) d Y^{i} d Y^{j}
$$

we also allow the dilaton $\Phi(Y)$ to depend on the transverse coordinates. We use $X^{\mu}$ for the noncompact dimensions and $Y^{i}$ for the transverse dimensions. The relevant terms in the world-sheet action for a $(p, q)$ string are

$$
S=-\frac{1}{2 \pi \alpha^{\prime}} \int d^{2} \sigma \nu\left(-\operatorname{det} h_{a b}\right)^{1 / 2}
$$

where

$$
\nu=\left(p^{2}+q^{2} e^{-2 \Phi(Y)}\right)^{1 / 2}, \quad h_{a b}=H^{-1 / 2}(Y) \eta_{\mu \nu} \partial_{a} X^{\mu} \partial_{b} X^{\nu}+H^{1 / 2}(Y) g_{i j}(Y) \partial_{a} Y^{i} \partial_{b} Y^{j}
$$

To obtain first the potential, we insert a static configuration $X^{0}=\sigma^{0}, X^{1}=\sigma^{1}$, $Y^{i}=$ constant. The action then reduces to a potential

$$
V(Y)=-\mathcal{L}=\frac{\nu(Y)}{2 \pi \alpha^{\prime} H^{1 / 2}(Y)} .
$$

Compactifications with string tensions below the Planck scale generally have branes and fluxes that produce a nontrivial warp factor and/or dilaton, so that the potential (17.4) 
depends nontrivially on the compact dimensions. The strings will then sit near the minimum of the potential. For strings that are supersymmetric with respect to all the branes the classical potential can cancel [4], and it will then be necessary to go to higher order or even to nonperturbative physics to find the leading effect.

Notice that if the dilaton is nontrivial then the position of the minimum will depend on $p$ and $q$. For the strongly warped geometries [6] which are our main focus, the variation of the dilaton is negligible. However, it should be noted that this effect has the possibility in principle to localize the different $(p, q)$ strings far enough apart that they will evolve as essentially independent networks. Roughly speaking they must be separated by more than a string length for this to happen; we will make a few further comments below.

To understand the fluctuations around the minima we will need to expand the action to second order in the $Y^{i}$. We choose coordinates such that the minimum is at $Y=0$ and $H^{1 / 2}(0) g_{i j}(0)=\delta_{i j}$. Then

$$
S \approx-\int d^{2} \sigma\left\{V(0)+\frac{1}{2} \partial_{i} \partial_{j} V(0) Y^{i} Y^{j}+\frac{\nu(0)}{4 \pi \alpha^{\prime}} \partial_{a} Y^{i} \partial^{a} Y^{i}\right\} .
$$

Notice that the $Y^{i}$ are now massive world-sheet fields. We are interested in the average spread of the $Y^{i}$. For a single scalar field with action

$$
S=-\frac{Z}{2} \int d^{2} \sigma\left(\partial_{a} \phi \partial^{a} \phi+m^{2} \phi^{2}\right)
$$

a Feynman diagram calculation gives

$$
\begin{aligned}
\left\langle\phi^{2}(0)\right\rangle & =\frac{1}{Z} \int^{\Lambda} \frac{d^{2} k}{(2 \pi)^{2}} \frac{1}{k^{2}+m^{2}} \\
& =\frac{1}{4 \pi Z} \ln \frac{\Lambda^{2}+m^{2}}{m^{2}} .
\end{aligned}
$$

In our case, the UV cutoff is the string scale. This is $\Lambda^{2} \sim 1 / \alpha^{\prime}$ as seen by a ten-dimensional observer, but in four-dimensional units this is redshifted to $\Lambda^{2} \sim$ $1 / \alpha^{\prime} H^{1 / 2}(0)$. Rotating coordinates to make $\partial_{i} \partial_{j} V(0)$ diagonal, we have

$$
\left\langle Y^{i} Y^{i}\right\rangle=\frac{\alpha^{\prime}}{2 \nu(0)} \omega_{i}, \quad \omega_{i}=\ln \left[1+\frac{\nu(0)}{2 \pi \alpha^{\prime 2} H^{1 / 2}(0) V_{, i i}(0)}\right] \quad(\text { no sum on } i)
$$

From this we learn that the fluctuations of the string in the transverse dimensions scale only logarithmically as we lower the scale of the potential that localizes the string; 
the linear scale of the fluctuations goes as the square root of the logarithm, and the volume goes as the cube of the logarithm. The fluctuation (7.8) is proportional to

$$
\nu(0)^{-1}=\frac{g_{\mathrm{s}}}{\left(p^{2} g_{\mathrm{s}}^{2}+q^{2}\right)^{1 / 2}},
$$

so for strings with D-brane charge it vanishes to leading order in perturbation theory.

The calculation that we have done is meaningful only when $\Lambda^{2} / \mathrm{m}^{2}$ is large, meaning that $V_{, i i}$ is small in string units. This corresponds to the geometry varying slowly on the string scale. ${ }^{12}$ In this case we would expect to be able to combine the flat spacetime calculation that we have previously done with an effective wavefunction for the string calculated as above. When $V_{, i i}$ is of order one in string units, so that there is no separation of scales, there is no way to use the flat spacetime calculation. It is then necessary to do a full perturbative string calculation in curved space, and for the present we can only guess at the magnitude of $P$. It is not clear whether there is any physical situation in which $V_{, i i}$ becomes much greater than one in string units, but if there is it will require some complementary method of calculation.

\subsection{Effect on reconnection}

The effective value of $1 / V_{\perp}$ is the density $\rho$ of the wavefunction where the strings coincide. For example, the effective density for an F-D collision is obtained from the field theory fluctuations for an F-string relative to a fixed center (since the fluctuations of the D-string are much smaller):

$$
\begin{aligned}
V_{\min } \rho_{\mathrm{FD}} & =V_{\min }\left\langle\delta^{6}(Y)\right\rangle \\
& =V_{\min } \int \frac{d^{6} l}{(2 \pi)^{6}}\left\langle e^{i l \cdot Y}\right\rangle \\
& =V_{\min } \int \frac{d^{6} l}{(2 \pi)^{6}} e^{-l_{i} l_{j}\left\langle Y^{i} Y^{j}\right\rangle / 2} \\
& =\frac{(4 \pi)^{3}}{\prod_{i} \omega_{i}^{1 / 2}} .
\end{aligned}
$$

This has the expected logarithmic behavior, with the coefficient of the logarithm now determined. This assumes that the F- and D-strings are localized at the same point.

\footnotetext{
${ }^{12} \mathrm{Also}$ in this limit the effective quartic coupling is small, so the only-loop calculation that we have done is valid.
} 
If the F-string potential is minimized at $Y=0$ and the D-string potential at $Y=Y_{\mathrm{D}}$ then

$$
\begin{aligned}
V_{\min } \rho_{\mathrm{FD}} & =V_{\min }\left\langle\delta^{6}\left(Y-Y_{\mathrm{D}}\right)\right\rangle \\
& =\frac{(4 \pi)^{3}}{\prod_{i} \omega_{i}^{1 / 2}} \exp \left[-\sum_{i} \frac{Y_{\mathrm{D}}^{i} Y_{\mathrm{D}}^{i}}{\alpha^{\prime} \omega_{i}}\right] .
\end{aligned}
$$

This illustrates the expected large suppression when the separation of the minima is larger than the string scale. For an F-F collision one has separate fields $Y$ and $Y^{\prime}$, and SO

$$
\begin{aligned}
V_{\min } \rho_{\mathrm{FF}} & =V_{\min }\left\langle\delta^{6}\left(Y-Y^{\prime}\right)\right\rangle \\
& =\frac{(2 \pi)^{3}}{\prod_{i} \omega_{i}^{1 / 2}} .
\end{aligned}
$$

Since the F-strings are of the same type their minima are coincident.

For the general $(p, q)-\left(p^{\prime}, q^{\prime}\right)$ collision the effect of a nonzero impact parameter $y$ is to replace

$$
x \rightarrow x e^{-y^{2} / 2 \pi \alpha^{\prime} \epsilon}
$$

in the general interaction probability (5.5). Let us apply this in the case (5.18) that only the bosonic tachyon and the massless fermions are important, as holds over most of the parameter space. Then

$$
1-P_{\mathrm{pp}}(0) \simeq \frac{\left(1-e^{-y^{2} / 2 \pi \alpha^{\prime} \epsilon}\right)^{4}}{1+e^{\theta / \epsilon} e^{-y^{2} / 2 \pi \alpha^{\prime} \epsilon}} .
$$

Again we see that for strings in different minima, the interaction probability falls rapidly for separations large compared to the string scale. For strings in the same minima, the quantum fluctuations (7.8) imply that we must average the result (7.14) over a gaussian wavefunction of this width.

A typical value of the correction factor is

$$
e^{-y^{2} / 2 \pi \alpha^{\prime} \epsilon} \sim \exp \left[-\frac{\sum_{i} \omega_{i}}{2 \pi \nu(0) \epsilon}\right]
$$

we have assumed strings of the same type, so summing fluctuations as in eq. (7.12) contributes a factor of 2 to the exponent. For D-strings, $\nu^{-1}(0)=g_{\mathrm{s}}$, so this is formally 
higher order in perturbation theory. However, the fluctuations in the different directions add to give an effective factor of 6 , and so for the typical $\epsilon \sim 0.3$ the exponent can be of order one if the logarithm $\omega_{i}$ is large. This would lift the suppression due to the fermion zero modes. The probability to produce tachyon string modes remains large until the suppression factor (7.15) approaches $e^{-\theta / \epsilon}$, but if the scale of $V_{, i i}$ is low this can be the case, at least for some range of angles. Thus there is the possibility that D-strings can pass through one another without reconnecting.

When the typical value of the exponent (7.15) becomes large, the dominant contribution to the reconnection probability comes from those collisions that happen to occur at small impact parameter, near the center of the gaussian distribution. In this case the reconnection probability is given by the ten-dimensional cross-section, which is given to reasonable approximation by the low-velocity limit (5.8), times the peak density

$$
\rho_{\mathrm{DD}}=\left.\rho_{\mathrm{FF}}\right|_{\omega_{i} \rightarrow g_{\mathrm{s}} \omega_{i}}
$$

the factor of $g_{\mathrm{s}}$ reflecting the smaller fluctuations of the heavier D-string.

\subsection{Model parameters}

\subsubsection{The IKLMT model}

In the $\mathbb{K L M T}$ model [6], inflation takes place in a highly warped throat whose local geometry is given by the Klebanov-Strassler solution [40]. The warp factor also produces a potential well for the transverse coordinates of the string. The geometry near the base of this solution is locally $\mathbb{R}^{3} \times S^{3}$,

$$
g_{i j}(Y) d Y^{i} d Y^{j}=d r^{2}+r^{2} d \Omega_{2}^{2}+R_{3}^{2} d \Omega_{3}^{2}
$$

where $R_{3}^{2}=b g_{\mathrm{s}} M \alpha^{\prime} ; M$ is an integer characterizing the number of flux units. The warp factor near the origin depends on the radial coordinate of $R^{3}$,

$$
H(Y)=H(0)\left(1-\frac{b^{\prime} r^{2}}{g_{\mathrm{s}} M \alpha^{\prime}}\right) .
$$

The energy scale of inflation in this model is of order $10^{-4}$ in Planck units, so $H^{-1 / 4}(0) \sim$ $10^{-4}$. The constants $b \approx b^{\prime} \approx 0.93$ [41] will be treated as 1 for our purposes. The dilaton in this solution is constant. There are also three- and five-form fluxes in the compact 
directions, but these do not enter into the string action. The product $g_{\mathrm{s}} M$ must be somewhat greater than one in order for the supergravity approximation to be valid.

This solution has the special property that the warp factor has its minimum not at a point but on the entire three-sphere at $r=0$. We first consider this geometry as it stands, and then consider corrections. The effective $V_{\perp}$ is given by combining the volume of the $S^{3}$ with the quantum fluctuations on the $R^{3}$,

$$
V_{\mathrm{min}} \rho_{\mathrm{FF}} \approx \frac{4 \pi}{\left(g_{\mathrm{s}} M\right)^{3 / 2}} \frac{(2 \pi)^{3 / 2}}{\ln ^{3 / 2}\left(1+g_{\mathrm{s}} M\right)} .
$$

Also, $\rho_{\mathrm{FD}}=2^{3 / 2} \rho_{\mathrm{FF}}$.

The fact that the potential is constant on the $S^{3}$ reflects an $S U(2) \times S U(2)$ symmetry of the Klebanov-Strassler solution. This local geometry is part of a larger Calabi-Yau solution, which can have no isometries. The breaking of the symmetry will generate an effective potential along the $S^{3}$, which will localize the strings. The warp factor $H^{-1 / 4}(0)$ is a measure of the size of the tip of the Klebanov-Strassler throat in terms of the underlying Calabi-Yau geometry [43]. It therefore governs the extent to which the throat feels the curvature of the geometry, and so size of the $S U(2) \times S U(2)$ breaking and the size of the potential. We will assume that the curvature of the Calabi-Yau manifold will have an effect on the throat geometry of order the warp factor squared, $H^{-1 / 2}(0) \sim 10^{-8}$, relative to the other scales in the throat, so that $\omega_{i} \sim \ln H^{1 / 2}(0)$ in the $S^{3}$ directions. $^{13}$ Then

$$
V_{\min } \rho_{\mathrm{FF}}=\frac{(2 \pi)^{3}}{\ln ^{3 / 2}\left(H^{1 / 2}(0)\right) \ln ^{3 / 2}\left(1+g_{\mathrm{s}} M\right)} .
$$

In this case $\rho_{\mathrm{FD}}$ contains an additional factor of 8 , but it may easily be the case that the $\mathrm{F}$ and D strings are localized at different points of the $S^{3}$, leading to the additional suppression (7.11).

The reader will notice that the density (17.20) might be less than the density (17.19), depending on the parameters. This is not a logical possibility. What is happening is that the fluctuations begin to fill out the whole $S^{3}$; thus, we should always use whichever of the densities (7.20, (7.19) is greater. In the fluctuation calculation, the log of the ratio of scales times the world-sheet coupling $1 / g_{\mathrm{s}} M$ is becoming large, and we must use the renormalization group to improve the calculation.

\footnotetext{
${ }^{13}$ A more complete analysis might give a different power of $H(0)$ inside the logarithm. This will affect some of the numerical estimates, but not the overall logic.
} 
For D-D collisions, the relevant quantity is

$$
\frac{y^{2}}{2 \pi \alpha^{\prime} \epsilon} \sim \inf \left[\frac{g_{\mathrm{s}} M}{2 \pi \epsilon}, \frac{3 g_{\mathrm{s}} \ln H(0)}{8 \pi \epsilon}\right],
$$

depending on whether the quantum fluctuations fill out the $S^{3}$. Again, the fermion zero modes are lifted to the extent that this is nonzero, and the tachyonic modes are not excited for collisions at angles less than $\epsilon \times(\mathbf{1 7 . 2 1})$.

\subsubsection{Large dimension models}

Now let us consider models in which $n$ dimensions have periodicity $2 \pi R$ and $6-n$ have the minimum periodicity $2 \pi \sqrt{\alpha^{\prime}}[42]$. The four- and ten-dimensional gravitational couplings are

$$
\kappa_{4}^{2}=\kappa^{2}(2 \pi R)^{-n}\left(2 \pi \sqrt{\alpha^{\prime}}\right)^{n-6} .
$$

It is convenient to rewrite this as

$$
G \mu_{\mathrm{D}}=\frac{g_{\mathrm{s}}}{16 \pi}\left(\frac{\alpha^{\prime}}{R^{2}}\right)^{n / 2}
$$

We will assume that the effects that fix the moduli and break supersymmetry produce modulations of the warp factor and/or the dilaton by a factor of order $\delta \lesssim 1$, as opposed to the large warping of the $\mathbb{K L M T}$ model. Then for F-strings, $V_{, i i} \sim \delta / 2 \pi \alpha^{\prime} R^{2}$ in the large directions, and so

$$
\begin{aligned}
\omega_{\text {large }} & \sim \ln \left(\frac{R^{2}}{\alpha^{\prime} \delta}\right) \\
& \sim \frac{2}{n} \ln \left(\frac{g_{\mathrm{s}}}{16 \pi G \mu_{\mathrm{D}} \delta}\right), \\
V_{\perp} \rho_{\mathrm{FF}} & =\left(2 \pi / \omega_{\text {large }}\right)^{n / 2} .
\end{aligned}
$$

In $\rho_{\mathrm{FD}}$ there is an additional $2^{n / 2}$ but the likelihood of a suppression factor from separated minima. For D-D collisions, the relevant quantity is

$$
\frac{y^{2}}{2 \pi \alpha^{\prime} \epsilon} \sim \frac{g_{\mathrm{s}}}{\pi \epsilon} \ln \left(\frac{g_{\mathrm{s}}}{16 \pi G \mu_{\mathrm{D}} \delta}\right) .
$$




\section{Final results}

Having assembled all of the relevant calculations, it is interesting now to insert some typical parameter values and obtain estimates for $P$. It is premature to take the details of these models too seriously, but it is important to get an idea of the range of possibilities - both as a guide for the network simulations needed to estimate signals, and also to get some sense of the extent to which we might be able to probe stringy physics by measuring the various intercommutation probabilities.

Consider first F-F reconnection. The reconnection probability is the earlier result (3.9) with one of eq. (7.19, 7.20, 17.24) in place of $V_{\min } / V_{\perp}$. We will denote these cases respectively as (A) $\mathbb{K L M T}$ averaged over the $S^{3}$, (B) $\mathbb{K L M T}$ with fluctuations that do not fill out the $S^{3}$, and (C) large dimensions. The function $f(v, \theta)$ is roughly 0.5 when averaged over angles and velocities. ${ }^{14}$ Then in the three cases

$$
\begin{aligned}
P_{\mathrm{FF}}(\mathrm{A}) & \sim \frac{100 g_{\mathrm{s}}^{1 / 2}}{M^{3 / 2} \ln ^{3 / 2}\left(1+g_{\mathrm{s}} M\right)}, \\
P_{\mathrm{FF}}(\mathrm{B}) & \sim \frac{1.5 g_{\mathrm{s}}^{2}}{\ln ^{3 / 2}\left(1+g_{\mathrm{s}} M\right)}, \\
P_{\mathrm{FF}}(\mathrm{C}) & \sim 0.5 g_{\mathrm{s}}^{2}\left[\frac{\pi n}{\ln \left(g_{\mathrm{s}} / 16 \pi G \mu_{\mathrm{D}} \delta\right)}\right]^{n / 2}
\end{aligned}
$$

In case $\mathrm{A}, P$ depends only weakly on $g_{\mathrm{s}}$ but strongly on $M$. The stability of these models requires $M$ to be at least 12, perhaps somewhat larger [44]. For $M=20$, gives $P \sim 0.25$ over the range of $g_{\mathrm{s}}$ between 1 and the GUT value 0.05, while for $M=100, P \sim 0.01$. Decreasing the value of $P$ increases the total density of string in the network roughly as $P^{-1}$, because the reconnection process is needed for long strings to decay. Thus, one might say that for $M=20$, it would be possible with precise observations and simulations to distinguish these strings from the gauge theory strings that have $P=1$, while for $M=100$ there would be a substantial enhancement of the string density. In case B, we should note that the supergravity approximation used in $\mathbb{K L M T}$ requires $g_{\mathrm{s}} M$ to be greater than one, but its logarithm need not be especially large. In this case, one has $P \lesssim g_{\mathrm{s}}^{2}$, so $P$ approaches one in the 'worst case' that $g_{\mathrm{s}} \sim 1$, and it is much less than one for $g_{\mathrm{s}}$ near the GUT value. Notice, following

\footnotetext{
${ }^{14}$ We have noted at the end of $\$ 5.2$ that at small velocities unitarization effects must reduce $P_{\mathrm{FF}}$. It is possible that these have some effect at the velocities relevant for cosmic strings.
} 
the discussion after eq. (7.20), that at any given point in $\mathbb{K} L \mathbb{M T}$ parameter space one should use whichever of $\mathrm{A}$ and $\mathrm{B}$ is larger. In the case $\mathrm{C}$, the scale $G \mu_{\mathrm{D}}$ is likely to lie between $10^{-6}$ and $10^{-12}[3,4]$. Then for $\delta=1$ and $n=2$, the factor in square brackets ranges between 0.3 and 1 so that $P$ is as in case $\mathrm{B}$ or perhaps an order of magnitude less. For $\delta=1$ and $n=6$, the factor in square brackets is between 1 and 3 and so $P$ is rather larger than in case $B$. If $\delta \ll 1$ then a further suppression is possible. It is worth noting that the reconnection probabilities found in $\S 7.2$ were enhanced by various powers of $2 \pi$, but that these are offset by the effects of the fluctuations in most cases.

For $\mathrm{F}-(p, q)$ reconnection, $P$ contains an extra factor of $2^{k / 2} / g_{\mathrm{s}}$, where $k=3,6, n$ respectively in cases A,B,C. Also the function $f$ is replaced with $h_{p, q}$ from eq. (4.9), which varies in roughly the same range as $f$ except for an extra factor of $q$. Thus the $\mathrm{F}-(p, q)$ reconnection probability is somewhat larger than the $\mathrm{F}-\mathrm{F}$ reconnection probability, if the strings are coincident in the transverse directions (when the perturbative calculation gives $P>1$ we assume that it is approaching saturation, $P \rightarrow 1$ ). If the strings sit at separated minima in the transverse directions, the $\mathrm{F}-(p, q)$ reconnection can be suppressed by an arbitrary amount, and can easily be negligible.

For the D-D reconnection probability, collecting together the results (17.21, 7.25) and inserting numerical values as above, we have (roughly)

$$
\frac{y^{2}}{2 \pi \alpha^{\prime} \epsilon} \sim \begin{cases}\mathrm{A}: & 0.5 g_{\mathrm{s}} M \\ \mathrm{~B}: & 15 g_{\mathrm{s}} \\ \mathrm{C}: & g_{\mathrm{s}}\left\{O(10 \text { to } 25)+\ln \left(g_{\mathrm{s}} / \delta\right)\right\}\end{cases}
$$

The multiplicative contribution of each would-be fermion zero mode to $\left(1-P_{\mathrm{pp}}\right)$ is $\left(1-e^{-y^{2} / 2 \pi \alpha^{\prime} \epsilon}\right)$, so we see that these zero modes are largely lifted in all cases, and for larger values of $g_{\mathrm{s}}$ (but still less than 1 ) the production of fermionic open strings is negligible. The contribution of the open string tachyons is $\left(1+e^{\theta / \epsilon} e^{-y^{2} / 2 \pi \alpha^{\prime} \epsilon}\right)$. Defining $y^{2} / 2 \pi \alpha^{\prime} \epsilon=g_{\mathrm{s}} K$, where we see that $K$ is a number of order 10 or more, the tachyon production is suppressed for $\theta<0.3 K g_{\mathrm{s}}$. Thus the suppression might be over a small range of angles or over all angles, depending on the precise values of $K$ and $g_{\mathrm{s}}$.

For $(p, q)-\left(p^{\prime}, q^{\prime}\right)$ collisions the result depends primarily on the values of $q$ and $q^{\prime}$. Making one or both of these larger than 1 enhances reconnection in two ways: the production of strings is enhanced by the Chan-Paton degeneracy, and the fluctuations are decreased due to the greater tension of the $(p, q)$ string. 
Note that while the naive interaction probability (5.18) for D-D collisions is close to one, we have identified two effects that can reduce it substantially: the need to produce $O\left(1 / g_{\mathrm{s}}\right)$ strings as discussed in $§ 5.5$, and the fluctuation effects considered here. We have seen that the former are effective only for $g_{\mathrm{s}}$ less than the GUT value 0.05, while the latter become most significant as $g_{\mathrm{s}} \rightarrow 1$; there may be some parameter values where both play a role.

The reader might be concerned that in our string calculations we have consistently worked to lowest order in $g_{\mathrm{s}}$, but that we have found that higher-order effects such as the D-string fluctuations can have an important effect. This is not inconsistent: the fluctuations are enhanced by large logarithms below the string scale, in the spirit of renormalization group calculations. Also, the D-D reconnection probability is parametrically $e^{-O\left(1 / g_{\mathrm{s}}\right)}$, but we have argued that the onset of this behavior is at a rather small value of $g_{\mathrm{s}}$.

\section{Conclusions}

For F-strings we have found reconnection probabilities in the range $10^{-3}$ to 1 , and for D-strings perhaps 0.1 to 1 . With sufficient precision these can be distinguished in most cases from gauge theory strings that have $P=1$ exactly.

If there are stable cosmic strings for more than one $(p, q)$ value, the reconnection probability for strings of different types plays an essential role. When this is large, there is the possibility that the strings freeze into a three-dimensional network that is very different from the usual scaling solution [45]. Whether this happens can only be determined by detailed simulations. When the different $(p, q)$ do not reconnect, the situation would seem to be simpler as the different networks evolve independently. However, it is not clear whether such independent networks ever form. The string network likely forms in a highly tangled state, which remains as the different string segments roll to their respective minima. As the network evolves, endpoint interactions allow the strings of different types to disconnect, and also allows the populations of the various $(p, q)$ values to change, as segments disappear and form, ${ }^{15}$ but it seems unlikely

\footnotetext{
${ }^{15}$ This also resolves the puzzle of what cuts off the values of $p$ and $q$ that are populated. One might expect that larger values are less likely to form, but the usual scaling solution is an attractor so very large values of $(p, q)$ might begin to scale after enough time. However, in the tangled network there will be some equilibration.
} 
that this is sufficiently efficient as to allow them to fully disconnect.

To summarize, the following would be interesting to simulate:

1. Networks of a single type of string, but with $P<1$. For simplicity one might initially ignore the $\theta$ and $v$ dependence and consider fixed $P$ down to around $10^{-3}$. The interesting question is how the various signals scale with $P$. Ultimately one might be able to detect the $\theta$ dependence from the spectrum of kink angles in the network, but this is far in the future.

2. Networks of $(p, q)$ strings, formed in an initially tangled state. Here the interesting question is whether the scaling regime is reached. For simplicity one might start by taking $P$ to be exactly one for strings of the same type, and either one or zero for strings of different types. For recent work see refs. [46].

Our study of string collisions has involved a wide range of interesting physics, both in the perturbative calculations of the reconnection process and in the effects of compactification. In the best case, where all the $(p, q)$ strings are cosmically stable, we might hope to see a great deal of string physics written in the sky.

\section{Acknowledgements}

We would like to thank Ed Copeland, Rob Myers, and Henry Tye for discussions. MJ and NJ would like to thank the KITP for hospitality and support through the Graduate Fellows program. This work was supported by National Science Foundation grants PHY99-07949, PHY00-98395, and PHY00-98631. 


\section{References}

[1] E. Witten, "Cosmic superstrings," Phys. Lett. B 153, 243 (1985).

[2] N. Jones, H. Stoica and S. H. Tye, "Brane interaction as the origin of inflation," JHEP 0207, 051 (2002) arXiv:hep-th/0203163.

[3] S. Sarangi and S. H. Tye, "Cosmic string production towards the end of brane inflation," Phys. Lett. B 536, 185 (2002) arXiv:hep-th/0204074.

[4] N. T. Jones, H. Stoica and S. H. Tye, "The production, spectrum and evolution of cosmic strings in brane inflation," Phys. Lett. B 563, 6 (2003) arXiv:hep-th/0303269.

[5] L. Pogosian, S. H. H. Tye, I. Wasserman and M. Wyman, "Observational constraints on cosmic string production during brane inflation," Phys. Rev. D 68, 023506 (2003) arXiv:hep-th/0304188.

[6] S. Kachru, R. Kallosh, A. Linde, J. Maldacena, L. McAllister and S. P. Trivedi, "Towards inflation in string theory," JCAP 0310, 013 (2003) arXiv:hep-th/0308055;

N. Iizuka and S. P. Trivedi, "An inflationary model in string theory," Phys. Rev. D 70, 043519 (2004) arXiv:hep-th/0403203.

[7] G. Dvali and A. Vilenkin, "Formation and evolution of cosmic D-strings," arXiv:hep-th/0312007.

[8] E. J. Copeland, R. C. Myers and J. Polchinski, "Cosmic F- and D-strings," arXiv:hep-th/0312067.

[9] L. Leblond and S. H. H. Tye, "Stability of D1-strings inside a D3-brane," arXiv:hep-th/0402072.

[10] M. B. Hindmarsh and T. W. Kibble, "Cosmic strings," Rept. Prog. Phys. 58, 477 (1995) arXiv:hep-ph/9411342;

A. Vilenkin and E.P.S. Shellard, Cosmic strings and other topological defects, Cambridge Univ. Press (Cambridge 1994).

[11] E. P. S. Shellard, "Cosmic String Interactions," Nucl. Phys. B 283, 624 (1987);

R.A. Matzner, "Interaction of $U(1)$ cosmic strings: numerical intercommutation," Comput. Phys. 2, 51 (1989);

K. J. M. Moriarty, E. Myers and C. Rebbi, J. Comput. Phys. 88, 467 (1990). 
[12] A. Vilenkin, "String dominated universe," Phys. Rev. Lett. 53, 1016 (1984); T. W. B. Kibble, "String dominated universe," Phys. Rev. D 33, 328 (1986).

[13] J. Polchinski, "Collision of macroscopic fundamental strings," Phys. Lett. B 209, 252 (1988).

[14] J. Dai and J. Polchinski, "The decay of macroscopic fundamental strings," Phys. Lett. B 220, 387 (1989).

[15] C. Bachas, "D-brane dynamics," Phys. Lett. B 374, 37 (1996) arXiv:hep-th/9511043.

[16] A. Hanany and K. Hashimoto, "Reconnection of colliding cosmic strings," JHEP 0506, 021 (2005) arXiv:hep-th/0501031.

[17] J. Polchinski, "Introduction to cosmic F- and D-strings," arXiv:hep-th/0412244.

[18] K. Hashimoto and D. Tong, "Reconnection of non-abelian cosmic strings," arXiv:hep-th/0506022.

[19] J. Preskill, "Vortices and Monopoles," in Architecture of the Fundamental Interactions at Short Distances, ed. P. Ramond and R. Stora, Amsterdam (NorthHolland, 1987), p. 235-338;

M. Sakellariadou and A. Vilenkin, "Cosmic-String Evolution In Flat Space-Time," Phys. Rev. D 42, 349 (1990);

"A note on the evolution of cosmic string / superstring networks," JCAP 0504, 003 (2005) arXiv:hep-th/0410234.

[20] D. Austin, E. J. Copeland and T. W. B. Kibble, "Evolution of cosmic string configurations," Phys. Rev. D 48, 5594 (1993) arXiv:hep-ph/9307325.

[21] A. Vilenkin and A. E. Everett, "Cosmic strings and domain walls in models with Goldstone and pseudogoldstone bosons," Phys. Rev. Lett. 48, 1867 (1982).

[22] J. Polchinski, "Dirichlet-branes and Ramond-Ramond charges," Phys. Rev. Lett. 75, 4724 (1995) arXiv:hep-th/9510017.

[23] J. F. G. Cascales, M. P. Garcia del Moral, F. Quevedo and A. M. Uranga, "Realistic D-brane models on warped throats: Fluxes, hierarchies and moduli stabilization," JHEP 0402, 031 (2004) arXiv:hep-th/0312051.

[24] J. A. Harvey and A. Strominger, "The heterotic string is a soliton," Nucl. Phys. B 449, 535 (1995) [Erratum-ibid. B 458, 456 (1996)] arXiv:hep-th/9504047; 
J. H. Schwarz, "An SL(2,Z) multiplet of type IIB superstrings," Phys. Lett. B 360, 13 (1995) [Erratum-ibid. B 364, 252 (1995)] arXiv:hep-th/9508143.

[25] E. Witten, "Bound states of strings and p-branes," Nucl. Phys. B 460, 335 (1996) arXiv:hep-th/9510135.

[26] J. H. Schwarz, "Lectures on superstring and M theory dualities," Nucl. Phys. Proc. Suppl. 55B, 1 (1997) arXiv:hep-th/9607201;

O. Aharony, J. Sonnenschein and S. Yankielowicz, "Interactions of strings and D-branes from M theory," Nucl. Phys. B 474, 309 (1996) arXiv:hep-th/9603009;

M. R. Gaberdiel and B. Zwiebach, "Exceptional groups from open strings," Nucl. Phys. B 518, 151 (1998) arXiv:hep-th/9709013;

O. Aharony, A. Hanany and B. Kol, "Webs of (p,q) 5-branes, five dimensional field theories and grid diagrams," JHEP 9801, 002 (1998) arXiv:hep-th/9710116;

K. Dasgupta and S. Mukhi, "BPS nature of 3-string junctions," Phys. Lett. B 423, 261 (1998) arXiv:hep-th/9711094;

A. Sen, "String network," JHEP 9803, 005 (1998) arXiv:hep-th/9711130;

S. J. Rey and J. T. Yee, "BPS dynamics of triple (p,q) string junction," Nucl. Phys. B 526, 229 (1998) arXiv:hep-th/9711202.

[27] J. Polchinski, String Theory. Vol. 2: Superstring Theory and Beyond. Cambridge Univ. Pr., 1998.

[28] C. G. Callan and R. R. Khuri, "A Comment On The Scattering Of Macroscopic Strings," Phys. Lett. B 261, 363 (1991);

R. R. Khuri, "Classical dynamics of macroscopic strings," Nucl. Phys. B 403, 335 (1993) arXiv:hep-th/9212029; "Veneziano amplitude for winding strings," Phys. Rev. D 48, 2823 (1993) arXiv:hep-th/9303074.

[29] D. Amati, M. Ciafaloni and G. Veneziano, "Superstring collisions at Planckian energies," Phys. Lett. B 197, 81 (1987);

I. J. Muzinich and M. Soldate, "High-energy unitarity of gravitation and strings," Phys. Rev. D 37, 359 (1988).

[30] D. J. Gross and P. F. Mende, "String Theory Beyond The Planck Scale," Nucl. Phys. B 303, 407 (1988);

P. F. Mende, "High-energy string collisions in a compact space," Phys. Lett. B 326, 216 (1994) arXiv:hep-th/9401126.

[31] E. S. Fradkin and A. A. Tseytlin, "Nonlinear electrodynamics from quantized strings," Phys. Lett. B 163, 123 (1985); 
C. G. Callan, C. Lovelace, C. R. Nappi and S. A. Yost, "String loop corrections to beta functions," Nucl. Phys. B 288, 525 (1987);

A. Abouelsaood, C. G. Callan, C. R. Nappi and S. A. Yost, "Open strings in background gauge fields," Nucl. Phys. B 280, 599 (1987);

N. Seiberg and E. Witten, "String theory and noncommutative geometry," JHEP 9909, 032 (1999) arXiv:hep-th/9908142.

[32] M. R. Garousi, "Superstring scattering from D-branes bound states," JHEP 9812, 008 (1998) arXiv:hep-th/9805078.

[33] G. Lifschytz, "Comparing D-branes to black-branes," Phys. Lett. B 388, 720 (1996) arXiv:hep-th/9604156.

[34] M. Berkooz, M. R. Douglas and R. G. Leigh, "Branes intersecting at angles," Nucl. Phys. B 480, 265 (1996) arXiv:hep-th/9606139.

[35] H. Arfaei and M. M. Sheikh Jabbari, "Different D-brane interactions," Phys. Lett. B 394, 288 (1997) arXiv:hep-th/9608167.

[36] J. S. Schwinger, "On gauge invariance and vacuum polarization," Phys. Rev. 82, 664 (1951).

[37] T. Banks and L. Susskind, "Brane - antibrane forces," arXiv:hep-th/9511194.

[38] A. Hashimoto and W. I. Taylor, "Fluctuation spectra of tilted and intersecting D-branes from the Born-Infeld action," Nucl. Phys. B 503, 193 (1997) arXiv:hep-th/9703217.

[39] A. V. Morosov, "Classical decay of a non-supersymmetric configuration of two D-branes," Phys. Lett. B 433, 291 (1998) arXiv:hep-th/9803110;

K. Hashimoto and S. Nagaoka, "Recombination of intersecting D-branes by local tachyon condensation," JHEP 0306, 034 (2003) arXiv:hep-th/0303204;

W. H. Huang, "Recombination of intersecting D-branes in tachyon field theory," Phys. Lett. B 564, 155 (2003) arXiv:hep-th/0304171;

N. T. Jones and S. H. H. Tye, "Spectral flow and boundary string field theory for angled D-branes," JHEP 0308, 037 (2003) arXiv:hep-th/0307092]. F. Epple and D. Lust, "Tachyon condensation for intersecting branes at small and large angles," Fortsch. Phys. 52, 367 (2004) arXiv:hep-th/0311182.

[40] I. R. Klebanov and M. J. Strassler, "Supergravity and a confining gauge theory: Duality cascades and $\chi$ SB-resolution of naked singularities," JHEP 0008, 052 (2000) arXiv:hep-th/0007191. 
[41] C. P. Herzog, I. R. Klebanov and P. Ouyang, "D-branes on the conifold and N = 1 gauge / gravity dualities," arXiv:hep-th/0205100.

[42] N. Arkani-Hamed, S. Dimopoulos and G. R. Dvali, "The hierarchy problem and new dimensions at a millimeter," Phys. Lett. B 429, 263 (1998) arXiv:hep-ph/9803315;

I. Antoniadis, N. Arkani-Hamed, S. Dimopoulos and G. R. Dvali, "New dimensions at a millimeter to a Fermi and superstrings at a TeV," Phys. Lett. B 436, 257 (1998) arXiv:hep-ph/9804398.

[43] S. B. Giddings, S. Kachru and J. Polchinski, "Hierarchies from fluxes in string compactifications," Phys. Rev. D 66, 106006 (2002) arXiv:hep-th/0105097.

[44] S. Kachru, R. Kallosh, A. Linde and S. P. Trivedi, "De Sitter vacua in string theory," Phys. Rev. D 68, 046005 (2003) arXiv:hep-th/0301240.

[45] T. W. B. Kibble, "Topology of cosmic domains and strings," J. Phys. A 9, 1387 (1976);

A. Vilenkin, "String dominated universe," Phys. Rev. Lett. 53, 1016 (1984).

[46] C. J. A. Martins, "Scaling laws for non-intercommuting cosmic string networks," Phys. Rev. D 70, 107302 (2004) arXiv:hep-ph/0410326;

S. H. Tye, I. Wasserman and M. Wyman, "Scaling of multi-tension cosmic superstring networks," Phys. Rev. D 71, 103508 (2005) [Erratum-ibid. D 71, 129906 (2005)] arXiv:astro-ph/0503506;

E. J. Copeland and P. M. Saffin, "On the evolution of cosmic-superstring networks," arXiv:hep-th/0505110. 